\title{
THE ECLIPSING BINARY V1061 CYGNI: CONFRONTING STELLAR EVOLUTION MODELS FOR ACTIVE AND INACTIVE SOLAR-TYPE STARS
}

\author{
Guillermo Torres, ${ }^{1}$ Claud H. Lacy, ${ }^{2}$ Laurence A. Marschall, ${ }^{3}$ Holly A. Sheets, ${ }^{4}$ and Jeff A. Mader ${ }^{5}$ \\ Received 2005 August 1; accepted 2005 November 30
}

\begin{abstract}
We present spectroscopic and photometric observations of the eclipsing system V1061 Cyg $(P=2.35$ days $)$. A third star is visible in the spectrum, and the system is a hierarchical triple. We combine the radial velocities for the three stars, times of eclipse, and intermediate astrometric data from the Hipparcos mission (abscissa residuals) to establish the elements of the outer orbit, which is eccentric and has a period of $15.8 \mathrm{yr}$. We determine accurate values for the masses, radii, and effective temperatures of the binary components: $M_{\mathrm{Aa}}=1.282 \pm 0.015 M_{\odot}, R_{\mathrm{Aa}}=$ $1.615 \pm 0.017 R_{\odot}$, and $T_{\text {eff }}^{\mathrm{Aa}}=6180 \pm 100 \mathrm{~K}$ for the primary (star Aa), and $M_{\mathrm{Ab}}=0.9315 \pm 0.0068 M_{\odot}, R_{\mathrm{Ab}}=$ $0.974 \pm 0.020 R_{\odot}$, and $T_{\text {eff }}^{\mathrm{Ab}}=5300 \pm 150 \mathrm{~K}$ for the secondary $(\mathrm{Ab})$. The mass of the tertiary is determined to be $M_{\mathrm{B}}=0.925 \pm 0.036 M_{\odot}$ and its effective temperature is $T_{\text {eff }}^{\mathrm{B}}=5670 \pm 150 \mathrm{~K}$. Current stellar evolution models agree well with the properties of the primary but show a very large discrepancy in the radius of the secondary, in the sense that the predicted values are $\sim 10 \%$ smaller than observed ( $\sim 5 \sigma$ effect). In addition, the temperature is cooler than predicted, by some $200 \mathrm{~K}$. These discrepancies are quite remarkable given that the star is only $7 \%$ less massive than the Sun, the calibration point of all stellar models. We identify the chromospheric activity as the likely cause of the effect. Inactive stars agree very well with the models, while active ones such as V1061 Cyg Ab appear systematically too large and too cool.
\end{abstract}

Subject headings: binaries: close — binaries: spectroscopic — stars: evolution — stars: individual (V1061 Cyg) — techniques: spectroscopic

Online material: color figures, machine-readable tables

\section{INTRODUCTION}

Accurately determined properties of stars in detached eclipsing binaries provide fundamental data for testing models of stellar structure and stellar evolution (see, e.g., Andersen 1991, 1997). For stars less massive than the Sun properties such as the stellar radius and the effective temperature have occasionally been found to disagree with model predictions (see, e.g., Lacy 1977; Popper 1997; Clausen et al. 1999a; Torres \& Ribas 2002; Ribas 2003). Directed efforts to find additional systems in this regime suitable for testing theory (Popper 1996; Clausen et al. 1999b) have produced a few cases, while other examples have been found serendipitously (e.g., Creevey et al. 2005; López-Morales \& Ribas 2005). The present binary system is in the second category, since it was originally thought to be of a completely different nature.

The photometric variability of V1061 Cygni (also known as HD 235444, HIP 104263, RX J2107.3+5202, $\alpha=21^{\mathrm{h}} 07^{\mathrm{m}} 20.52$, $\delta=+52^{\circ} 02^{\prime} 58^{\prime \prime} .4$ [J2000.0], spectral type F9, $V=9.24$ ) was discovered photographically by Strohmeier \& Knigge (1959), and the object was classified by Strohmeier et al. (1962) as an Algoltype binary with a period of 2.346656 days. Other than occasional measurements of the time of primary eclipse, the system received very little attention until the spectroscopic work by Popper (1996), who observed it as part of his program to search for eclipsing

\footnotetext{
1 Harvard-Smithsonian Center for Astrophysics, 60 Garden Street, Cambridge, MA 02138; gtorres@cfa.harvard.edu.

2 Department of Physics, University of Arkansas, 226 Physics Building, Fayetteville, AR 72701; clacy@uark.edu.

3 Department of Physics, Gettysburg College, 300 North Washington Street, Gettysburg, PA 17325; marschal@gettysburg.edu.

4 Department of Physics and Astronomy, Dartmouth College, 6127 Wilder Laboratory, Hanover, NH 03755; holly.a.sheets@dartmouth.edu.

5 W. M. Keck Observatory, 65-1120 Mamalahoa Highway, Kamuela, HI 96743; jmader@keck.hawaii.edu.
}

binaries containing at least one lower main-sequence star (late $\mathrm{F}$ to $\mathrm{K}$ ). On the basis of two high-resolution spectra and other information Popper concluded that V1061 Cyg was most likely a semidetached system of the rare "cool Algol" class and dropped it from his program. Unlike the classical Algols, which are composed of a cool giant or subgiant and an early-type star, the mass gainer in the cool Algols is also of late spectral type (see Popper 1992). Since less than a dozen of these systems are known, V1061 Cyg was placed on the observing list at the Harvard-Smithsonian Center for Astrophysics (CfA) in 1998 for spectroscopic monitoring, and photometric observations began later. Not only did we discover that it is not a cool Algol (it is well detached, as reported by Sheets et al. 2003), but we also found that (1) it is triple lined (and a hierarchical triple), (2) the secondary in the eclipsing pair is less massive than the Sun and therefore potentially interesting for constraining models of stellar structure and evolution (Popper's original motivation for observing it), and (3) the mass ratio of the binary is quite different from unity, which makes it a favorable case for such tests. Furthermore, the comparison with theory shows a significant discrepancy in the radius of the secondary, corroborating similar evidence from other systems and providing some insight into the problem.

We describe below our observations and complete analysis of this system, including a discussion of the possible nature of the deviations from the models for low-mass stars.

\section{OBSERVATIONS AND REDUCTIONS}

\subsection{Spectroscopy}

V1061 Cyg was observed at the CfA with an echelle spectrograph on the $1.5 \mathrm{~m}$ Wyeth reflector at the Oak Ridge Observatory (eastern Massachusetts) and occasionally also with a nearly identical instrument on the $1.5 \mathrm{~m}$ Tillinghast reflector at the F. L. Whipple 

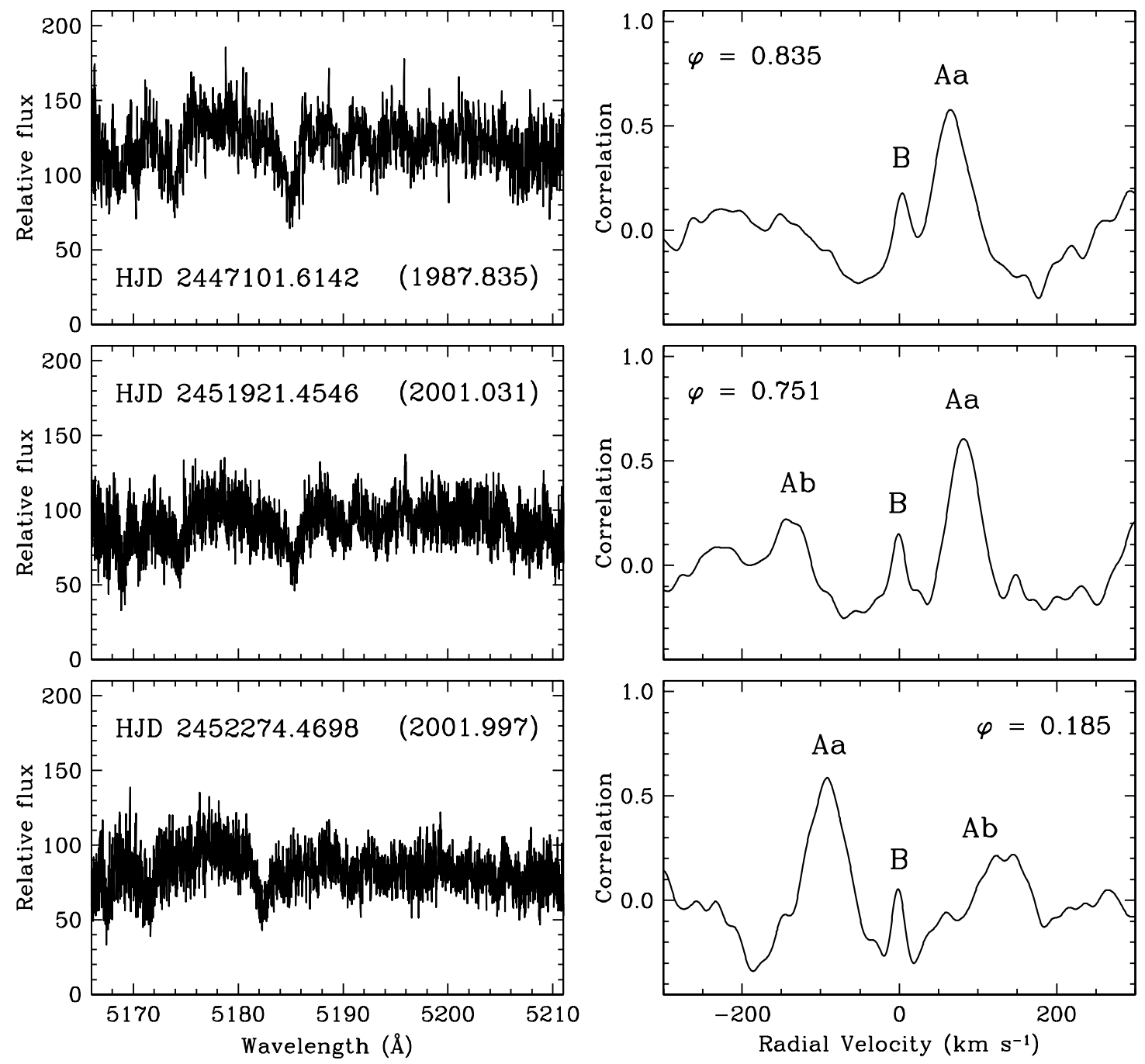

FIG. 1.-Sample spectra of V1061 Cyg (left) and corresponding cross-correlation functions (right) showing a broad-lined (Aa) and a sharp-lined (B) component. The Julian date, year, and orbital phase $(\varphi)$ are indicated. The tertiary star (Ab) is not obvious in the 1987 spectrum but is in many of the more recent observations.

Observatory (Arizona). A single echelle order was recorded using intensified photon-counting Reticon detectors, spanning about $45 \AA$ at a central wavelength of $5187 \AA$, which includes the $\mathrm{Mg}_{\mathrm{I}} b$ triplet. The resolving power of these instruments is $\lambda / \Delta \lambda \approx$ 35,000 , and the signal-to-noise ratios ( $\mathrm{S} / \mathrm{Ns}$ ) achieved range from 13 to about 40 per resolution element of $8.5 \mathrm{~km} \mathrm{~s}^{-1}$. A total of 74 spectra were collected from 1998 August until 2005 April. One archival spectrum from 1987 obtained with the same instrumentation was used as well. That observation was taken by J. Andersen, possibly at the request of D. Popper (J. Andersen 2005, private communication), and it shows double lines (one sharp and one broad; see Fig. 1). Popper (1996) referred to that observation and remarked that his own two spectra of the star also showed a sharplined and a broad-lined component. Most of our more recent observations display similar features. The fact that the sharp-lined star is hardly moving whereas the other one moves significantly led Popper to infer a mass ratio of roughly $8: 1$ and to conclude that V1061 Cyg is a cool Algol.

As it turns out, the sharp-lined component is not the secondary in the eclipsing binary. Closer inspection of the cross-correlation functions, after we had estimated crude velocities and discovered that the sharp-lined and broad-lined stars were not moving together, revealed the presence of a third star (the true secondary) changing velocity with the same 2.35 day period as the broadlined component. This is illustrated in Figure 1. In the following we refer to the broad-lined primary of the eclipsing binary (which is the more massive one of the pair) as star Aa, to the secondary as star $\mathrm{Ab}$, and to the tertiary as star $\mathrm{B}$.

Radial velocities for the three stars were derived using an extension of the two-dimensional cross-correlation algorithm TODCOR (Zucker \& Mazeh 1994) to three dimensions (Zucker et al. 1995). This technique uses three templates, one for each star, and is well suited to our relatively low $\mathrm{S} / \mathrm{N}$ spectra, allowing velocities to be obtained reliably even when the spectral lines are blended. Templates for the cross-correlations were selected from an extensive library of calculated spectra based on model atmospheres by R. L. Kurucz, ${ }^{6}$ computed for us by J. Morse (see also Nordström et al. 1994; Latham et al. 2002). These calculated spectra are available for a wide range of effective temperatures $\left(T_{\text {eff }}\right)$, projected rotational velocities $(v \sin i)$, surface gravities $(\log g)$, and metallicities. Experience has shown that radial velocities are largely insensitive to the surface gravity and metallicity adopted for the templates. Consequently, the optimum template for each star was determined from grids of cross-correlations over broad ranges in temperature and rotational velocity (since these are the parameters that affect the radial velocities the most), seeking to maximize the average correlation weighted by the strength of each exposure. Surface gravities of $\log g=4.0$ and 4.5 were adopted for Aa and

\footnotetext{
${ }^{6}$ Available at http://cfaku5.cfa.harvard.edu.
} 
$\mathrm{Ab}$, from the light-curve analysis described in $\S 2.3$, and for star B we adopted $\log g=4.5$. Solar metallicity was assumed throughout, which the discussion in $\S 5$ shows to be a good assumption in this case. The procedure of optimizing the templates leads to best-fit parameters for stars $\mathrm{Aa}, \mathrm{Ab}$, and $\mathrm{B}$, respectively, as follows:

$$
\begin{gathered}
T_{\mathrm{eff}}=6160 \pm 100 \mathrm{~K}, \quad v \sin i=36 \pm 2 \mathrm{~km} \mathrm{~s}^{-1} \\
T_{\mathrm{eff}}=5400 \pm 200 \mathrm{~K}, \quad v \sin i=20 \pm 3 \mathrm{~km} \mathrm{~s}^{-1} \\
T_{\mathrm{eff}}=5670 \pm 150 \mathrm{~K}, \quad v \sin i=2 \pm 3 \mathrm{~km} \mathrm{~s}^{-1}
\end{gathered}
$$

The stability of the zero point of our velocity system was monitored by means of exposures of the dusk and dawn sky, and small run-to-run corrections were applied in the manner described by Latham (1992). In addition to the radial velocities, we derived the light contributions of each star following Zucker et al. (1995). These are $l_{\mathrm{Aa}}=0.75 \pm 0.01, l_{\mathrm{Ab}}=0.09 \pm 0.01$, and $l_{\mathrm{B}}=0.16 \pm 0.01$, expressed as fractions of the total light, and they correspond to the mean wavelength of our observations (5187 ̊).

Due to the narrow wavelength coverage of the CfA spectra, there is always the possibility of systematic errors in the velocities, resulting from lines of the stars moving in and out of the window with orbital phase (Latham et al. 1996). Occasionally these errors are significant, and experience has shown that this must be checked on a case-by-case basis (see, e.g., Torres et al. 1997, 2000). For this we performed numerical simulations in which we generated artificial composite spectra by adding together synthetic spectra for the three components, with Doppler shifts appropriate for each actual time of observation, computed from a preliminary orbital solution. The light fractions adopted were those derived above. We then processed these simulated spectra with the three-dimensional version of TODCOR in the same manner as the real spectra and compared the input and output velocities. The differences were typically small for stars Aa and $\mathrm{B}$ (under $1 \mathrm{~km} \mathrm{~s}^{-1}$ ) and were up to about $3 \mathrm{~km} \mathrm{~s}^{-1}$ for star $\mathrm{Ab}$. We applied these differences as corrections to the raw velocities, although they made little difference in the results other than slightly decreasing the residuals from the orbital fits. The final velocities including these corrections are given in Table 1. Similar corrections were derived for the light fractions and are already accounted for in the values reported above. As a further test we changed the template parameters for the two fainter stars, which are more uncertain, and recomputed the radial velocities and corrections. The resulting velocities were hardly different, giving us confidence in the robustness of the procedures.

A double-lined spectroscopic orbital solution for the $\mathrm{Aa}+\mathrm{Ab}$ system (see the second column of Table 10 below) produced a reasonably good fit with rms residuals of 2.4 and $5.1 \mathrm{~km} \mathrm{~s}^{-1}$, at about the level expected based on the broad lines and faint secondary. However, the residuals showed an obvious downward trend, less obvious but still present also in the secondary. The velocities for star B show a drift in the opposite direction (Fig. 2) with a slope about twice as steep, or $\sim 5 \mathrm{~km} \mathrm{~s}^{-1}$ per 1000 days. The system is thus a hierarchical triple, with a period for the outer orbit that could be very long, judging from the data available. The spectroscopic material alone does not sufficiently constrain the wide orbit to properly account for the change in the center-of-mass velocity of the eclipsing pair, which could introduce biases in the derived masses of components $\mathrm{Aa}$ and $\mathrm{Ab}$. However, as we show in the next section, the additional information provided by the measurement of times of eclipse and, to some extent, the astrometric data (ESA 1997) from the Hipparcos mission described later are very helpful in constraining the elements of the outer orbit.

\subsection{Times of Eclipse}

Measurements of the times of minimum light for V1061 Cyg spanning more than seven decades have been made by photographic, visual, and photoelectric/CCD techniques. Table 2 collects all available estimates, including some that have not appeared previously in print and were kindly communicated to us by J. M. Kreiner (see Kreiner et al. 2001). A total of 43 timings for the deeper primary minimum and six for the secondary are listed. Many of the more recent measurements between 2001 and 2004 are based on our own photometric observations with a robotic telescope described in more detail below. The $O-C$ residuals from a linear ephemeris are shown in Figure 3 and exhibit a pattern of variations caused by the presence of the distant third component (light-travel effect). This is most obvious in the more accurate and numerous measurements of the last 25 years, during which more than a full cycle has been covered. The period of the outer orbit is therefore fairly well constrained by these data, as is the amplitude of the variation, which is directly related to the velocity amplitude of the binary around the center of mass of the triple. These measurements provide information complementary to that from the radial velocities, and we incorporate them into the analysis in $\S 3$.

\subsection{Photometry}

Absolute photometry of V1061 Cyg in the $U B V$ system was obtained by Lacy (1992). The value of the reddening-free parameter $Q=(U-B)-0.72(B-V)$ was calculated and compared with the standard-star values of Johnson \& Morgan (1953). The value corresponded to that of a spectral type G0 star with an intrinsic color of $(B-V)_{0}=0.60_{-0.10}^{+0.00}$, with no significant interstellar reddening. The uncertainty here comes mainly from the calibration. The observed mean color index corresponds to a spectral type F9 star with a temperature of $6040 \mathrm{~K}$, according to the calibration of Popper (1980). After some iterations with the photometric light-curve fitting algorithm described below, it was found that the visual surface brightness ratio $J_{\mathrm{Ab}} / J_{\mathrm{Aa}}$ is about 0.43 , which constrains the difference in the visual flux parameter $F_{V}^{\prime}$ and hence the temperature difference through the calibration in Table 1 of Popper (1980). This procedure leads to photometric temperature estimates of $6200 \pm 100 \mathrm{~K}$ for the primary and $5280 \pm 100 \mathrm{~K}$ for the secondary component. These temperatures were used to estimate the limb-darkening and gravitybrightening parameters to be used in the photometric modeling. They are in excellent agreement with those of the spectroscopic analysis above.

Relative photometric observations of V1061 Cyg were obtained at two different facilities. Measurements in the $V$ band were made with the $0.26 \mathrm{~m}$ URSA robotic telescope at Kimpel Observatory on the campus of the University of Arkansas at Fayetteville. A total of 6914 observations were collected between 2001 March and 2004 October, which are given in Table 3. For details on the observation and reduction methods the reader is referred to the description by Lacy et al. (2004). The precision of an individual measurement is estimated to be 0.010 mag. Observations were also made with the $0.4 \mathrm{~m} \mathrm{f} / 11$ Ealing Cassegrain reflector at Gettysburg College Observatory, in Gettysburg (Pennsylvania). The detector was a Photometrics (Roper Scientific) $\mathrm{CH}-350$ thermoelectrically cooled CCD camera with a back-illuminated SITe 003B $1024 \times 1024$ chip and standard Bessell BVRI filters. Tables 4-7 list the observations obtained between 2002 
TABLE 1

Radial Velocity Measurements for V1061 Cyg

\begin{tabular}{|c|c|c|c|c|c|c|c|c|c|c|}
\hline $\begin{array}{c}\text { HJD } \\
(2,400,000+)\end{array}$ & Year & $\begin{array}{c}\mathrm{RV}_{\mathrm{Aa}} \\
\left(\mathrm{km} \mathrm{s}^{-1}\right)\end{array}$ & $\begin{array}{c}\mathrm{RV}_{\mathrm{Ab}} \\
\left(\mathrm{km} \mathrm{s}^{-1}\right)\end{array}$ & $\begin{array}{c}\mathrm{RV}_{\mathrm{B}} \\
\left(\mathrm{km} \mathrm{s}^{-1}\right)\end{array}$ & $\begin{array}{c}(O-C)_{\mathrm{Aa}} \\
\left(\mathrm{km} \mathrm{s}^{-1}\right)\end{array}$ & $\begin{array}{c}(O-C)_{\mathrm{Ab}} \\
\left(\mathrm{km} \mathrm{s}^{-1}\right)\end{array}$ & $\begin{array}{l}(O-C)_{\mathrm{B}} \\
\left(\mathrm{km} \mathrm{s}^{-1}\right)\end{array}$ & Inner Phase ${ }^{a}$ & Outer Phase $^{a}$ & $\begin{array}{c}\Delta T^{\mathrm{b}} \\
\text { (days) }\end{array}$ \\
\hline $47,101.6142 .$. & 1987.8347 & +66.13 & -106.02 & +2.24 & -0.13 & +7.64 & -1.13 & 0.8346 & 0.7504 & -0.0045 \\
\hline $51,039.8277 \ldots$ & 1998.6169 & -35.07 & +30.15 & -7.37 & -0.30 & -5.64 & -0.27 & 0.0549 & 0.4313 & -0.0143 \\
\hline $51,057.7385 \ldots \ldots$ & 1998.6660 & +74.19 & -119.55 & -8.69 & -1.80 & -2.80 & -1.69 & 0.6874 & 0.4343 & -0.0143 \\
\hline $51,075.7492 \ldots$ & 1998.7153 & -71.66 & +84.41 & -5.67 & +0.32 & -2.39 & +1.22 & 0.3624 & 0.4375 & -0.0144 \\
\hline $51,087.6477 \ldots \ldots$ & 1998.7478 & -40.88 & +42.78 & -5.91 & +0.30 & -1.56 & +0.91 & 0.4328 & 0.4395 & -0.0144 \\
\hline $51,089.6402 \ldots \ldots$ & 1998.7533 & -90.48 & +115.25 & -6.76 & +0.79 & +1.99 & +0.05 & 0.2819 & 0.4399 & -0.0144 \\
\hline 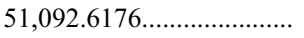 & 1998.7614 & +22.87 & -41.20 & -5.72 & +0.59 & +1.83 & +1.07 & 0.5507 & 0.4404 & -0.0144 \\
\hline 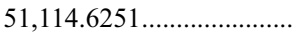 & 1998.8217 & +31.92 & -58.71 & -4.83 & -0.77 & -1.23 & +1.84 & 0.9289 & 0.4442 & -0.0144 \\
\hline $51,122.6356 \ldots \ldots$ & 1998.8436 & -77.41 & +105.84 & -5.94 & +1.28 & +10.08 & +0.68 & 0.3425 & 0.4456 & -0.0144 \\
\hline 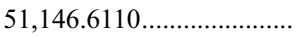 & 1998.9093 & +25.53 & -40.99 & -6.31 & -1.10 & +8.35 & +0.17 & 0.5593 & 0.4497 & -0.0145 \\
\hline $51,163.5148 \ldots \ldots$ & 1998.9556 & +83.04 & -121.98 & -5.20 & +0.87 & +3.89 & +1.18 & 0.7627 & 0.4526 & -0.0145 \\
\hline 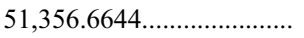 & 1999.4844 & -42.89 & +46.35 & -4.87 & +0.90 & -0.04 & +0.40 & 0.0711 & 0.4860 & -0.0145 \\
\hline $51,423.5273 \ldots$. & 1999.6674 & +28.65 & -48.61 & -5.95 & +0.28 & +4.70 & -1.06 & 0.5640 & 0.4976 & -0.0145 \\
\hline $51,464.6818 \ldots \ldots$ & 1999.7801 & -56.90 & +74.95 & -5.01 & +1.50 & +9.06 & -0.35 & 0.1015 & 0.5047 & -0.0144 \\
\hline $51,473.6526 \ldots \ldots$ & 1999.8047 & +32.28 & -64.30 & -5.45 & -1.79 & -2.87 & -0.84 & 0.9244 & 0.5063 & -0.0144 \\
\hline $51,477.5968 \ldots \ldots$ & 1999.8155 & +48.61 & -77.00 & -3.19 & +0.85 & +3.29 & +1.40 & 0.6051 & 0.5069 & -0.0144 \\
\hline 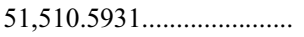 & 1999.9058 & +69.96 & -115.42 & -4.44 & +0.25 & -4.73 & -0.04 & 0.6661 & 0.5126 & -0.0143 \\
\hline $51,537.4847 \ldots \ldots$. & 1999.9794 & -68.89 & +85.11 & -3.12 & -0.24 & +5.52 & +1.13 & 0.1257 & 0.5173 & -0.0143 \\
\hline 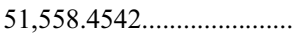 & 2000.0368 & -38.41 & +40.50 & -3.26 & +1.08 & +1.16 & +0.87 & 0.0616 & 0.5209 & -0.0143 \\
\hline 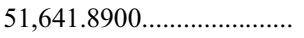 & 2000.2653 & +51.05 & -85.08 & -2.42 & -1.32 & +2.47 & +1.24 & 0.6169 & 0.5353 & -0.0140 \\
\hline 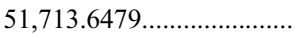 & 2000.4617 & -89.95 & +99.16 & -3.43 & -0.48 & -8.10 & -0.17 & 0.1958 & 0.5477 & -0.0138 \\
\hline $51,715.6836 \ldots \ldots \ldots \ldots \ldots \ldots \ldots \ldots \ldots \ldots \ldots \ldots$ & 2000.4673 & -40.85 & +37.33 & -2.04 & -0.13 & -2.83 & +1.21 & 0.0633 & 0.5481 & -0.0138 \\
\hline 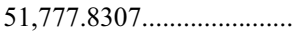 & 2000.6375 & +14.28 & -33.17 & -3.10 & -4.29 & +8.63 & -0.20 & 0.5467 & 0.5588 & -0.0136 \\
\hline 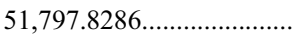 & 2000.6922 & -43.62 & +35.79 & -2.25 & -0.04 & -7.84 & +0.54 & 0.0686 & 0.5623 & -0.0135 \\
\hline $51,842.6036 \ldots \ldots$ & 2000.8148 & -77.47 & +94.87 & -1.64 & +0.26 & +4.49 & +0.90 & 0.1490 & 0.5700 & -0.0133 \\
\hline $51,857.6308 \ldots \ldots$ & 2000.8559 & +21.79 & -42.67 & -1.56 & +0.23 & +3.68 & +0.89 & 0.5527 & 0.5726 & -0.0133 \\
\hline $51,861.5475 \ldots \ldots$. & 2000.8667 & -93.89 & +112.74 & -3.22 & -0.41 & +0.79 & -0.79 & 0.2218 & 0.5733 & -0.0132 \\
\hline $51,888.5267 \ldots \ldots$ & 2000.9405 & +79.34 & -120.56 & -3.76 & +0.30 & +5.08 & -1.48 & 0.7187 & 0.5780 & -0.0131 \\
\hline $51,902.4960 \ldots \ldots$ & 2000.9788 & +70.32 & -112.81 & -2.21 & +0.06 & +0.83 & -0.01 & 0.6716 & 0.5804 & -0.0130 \\
\hline 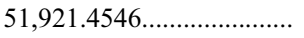 & 2001.0307 & +80.78 & -129.06 & -2.76 & +0.13 & -1.02 & -0.67 & 0.7507 & 0.5837 & -0.0130 \\
\hline $52,071.7515 \ldots \ldots \ldots \ldots \ldots \ldots \ldots \ldots \ldots \ldots \ldots$ & 2001.4422 & +78.34 & -115.61 & -1.55 & +2.05 & +7.26 & -0.30 & 0.7983 & 0.6097 & -0.0121 \\
\hline 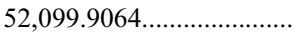 & 2001.5193 & +76.42 & -117.95 & -1.80 & -0.13 & +5.44 & -0.71 & 0.7963 & 0.6145 & -0.0119 \\
\hline $52,105.5866 \ldots \ldots$. & 2001.5348 & -92.25 & +104.57 & -0.43 & +1.28 & -6.09 & +0.63 & 0.2168 & 0.6155 & -0.0119 \\
\hline 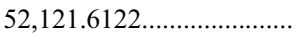 & 2001.5787 & -31.49 & +36.17 & +1.78 & +1.19 & +9.35 & +2.75 & 0.0460 & 0.6183 & -0.0118 \\
\hline $52,158.5190 \ldots \ldots \ldots \ldots \ldots \ldots \ldots \ldots$ & 2001.6797 & +77.10 & -129.27 & -0.11 & -2.04 & -1.99 & +0.65 & 0.7735 & 0.6247 & -0.0116 \\
\hline $52,182.6433 \ldots \ldots \ldots \ldots \ldots \ldots \ldots . .$. & 2001.7458 & -36.58 & +34.81 & -0.92 & +0.38 & +2.44 & -0.29 & 0.0539 & 0.6288 & -0.0114 \\
\hline $52,194.6505 \ldots \ldots$ & 2001.7786 & -83.42 & +109.65 & -0.30 & +1.53 & +11.30 & +0.26 & 0.1707 & 0.6309 & -0.0113 \\
\hline $52,220.6532 \ldots \ldots$ & 2001.8498 & -97.12 & +112.70 & -0.65 & -1.43 & -0.30 & -0.24 & 0.2515 & 0.6354 & -0.0111 \\
\hline $52,226.5786 \ldots \ldots$ & 2001.8661 & +78.36 & -127.09 & -0.74 & -0.36 & -0.01 & -0.36 & 0.7766 & 0.6364 & -0.0111 \\
\hline $52,234.5686 \ldots \ldots$ & 2001.8879 & -87.85 & +98.85 & -1.62 & -0.15 & -3.07 & -1.29 & 0.1814 & 0.6378 & -0.0110 \\
\hline $52,240.5913 \ldots \ldots$ & 2001.9044 & +77.39 & -128.49 & +1.00 & -2.51 & +0.29 & +1.30 & 0.7479 & 0.6388 & -0.0110 \\
\hline 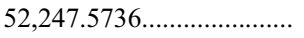 & 2001.9235 & +79.01 & -123.51 & -0.23 & +0.35 & +3.62 & +0.03 & 0.7234 & 0.6401 & -0.0109 \\
\hline $52,266.4777 \ldots \ldots \ldots \ldots \ldots \ldots \ldots$ & 2001.9753 & +78.62 & -130.69 & -1.33 & +0.25 & -3.87 & -1.18 & 0.7792 & 0.6433 & -0.0108 \\
\hline $52,274.4698 \ldots \ldots \ldots \ldots \ldots \ldots \ldots \ldots \ldots \ldots$ & 2001.9972 & -91.28 & +103.79 & -1.42 & -2.68 & +0.86 & -1.31 & 0.1850 & 0.6447 & -0.0107 \\
\hline $52,287.4668 \ldots \ldots \ldots \ldots \ldots \ldots \ldots \ldots$ & 2002.0328 & +78.79 & -123.26 & -1.55 & +0.20 & +3.98 & -1.52 & 0.7236 & 0.6470 & -0.0106 \\
\hline $52,416.7769 \ldots \ldots \ldots \ldots \ldots \ldots \ldots \ldots$ & 2002.3868 & +67.27 & -115.52 & +0.12 & -1.87 & -0.55 & -0.58 & 0.8280 & 0.6693 & -0.0095 \\
\hline 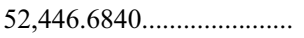 & 2002.4687 & +29.45 & -61.17 & -0.44 & -0.89 & +0.56 & -1.31 & 0.5727 & 0.6745 & -0.0092 \\
\hline $52,458.6699 \ldots \ldots$ & 2002.5015 & +72.05 & -117.36 & +3.17 & +0.92 & +0.58 & +2.23 & 0.6804 & 0.6766 & -0.0091 \\
\hline $52,537.6959 \ldots \ldots \ldots \ldots \ldots \ldots \ldots \ldots$ & 2002.7179 & -78.33 & +90.48 & +2.81 & -0.91 & +4.42 & +1.42 & 0.3568 & 0.6902 & -0.0084 \\
\hline $52,538.7321 \ldots \ldots$ & 2002.7207 & +76.54 & -122.17 & -0.43 & +1.36 & +1.80 & -1.82 & 0.7983 & 0.6904 & -0.0084 \\
\hline $52,566.6749 \ldots \ldots$ & 2002.7972 & +74.98 & -123.61 & +2.60 & -0.81 & +1.36 & +1.05 & 0.7060 & 0.6952 & -0.0081 \\
\hline $52,613.5082 \ldots \ldots$ & 2002.9254 & +66.53 & -112.28 & +1.19 & +0.13 & +0.03 & -0.63 & 0.6636 & 0.7033 & -0.0076 \\
\hline $52,626.4669 \ldots \ldots \ldots \ldots \ldots \ldots \ldots \ldots$ & 2002.9609 & -89.56 & +100.34 & +1.01 & +0.07 & -2.02 & -0.88 & 0.1859 & 0.7056 & -0.0075 \\
\hline $52,638.5178 \ldots \ldots$ & 2002.9939 & -89.41 & +94.18 & +1.77 & -1.39 & -5.90 & -0.19 & 0.3213 & 0.7076 & -0.0074 \\
\hline $52,651.4778 \ldots \ldots \ldots \ldots \ldots \ldots \ldots \ldots$ & 2003.0294 & +63.56 & -112.08 & +1.00 & -0.46 & -2.84 & -1.03 & 0.8441 & 0.7099 & -0.0072 \\
\hline $52,750.8421 \ldots \ldots \ldots \ldots \ldots \ldots \ldots$ & 2003.3014 & -88.59 & +105.04 & +2.82 & +1.69 & +2.49 & +0.22 & 0.1875 & 0.7271 & -0.0061 \\
\hline $52,788.7918 \ldots \ldots \ldots \ldots \ldots \ldots \ldots \ldots$ & 2003.4053 & -77.02 & +92.33 & +6.46 & +0.03 & +8.21 & +3.65 & 0.3596 & 0.7336 & -0.0057 \\
\hline $52,797.7304 \ldots \ldots \ldots \ldots \ldots \ldots \ldots \ldots$ & 2003.4298 & -89.02 & +96.14 & +5.91 & -3.17 & -0.06 & +3.05 & 0.1687 & 0.7352 & -0.0056 \\
\hline $52,891.7370 \ldots \ldots \ldots \ldots \ldots \ldots \ldots \ldots \ldots$ & 2003.6872 & -101.07 & +113.83 & +2.46 & -4.54 & +3.47 & -0.93 & 0.2290 & 0.7514 & -0.0044 \\
\hline 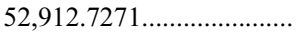 & 2003.7446 & -88.21 & +100.85 & +3.76 & -0.74 & +3.07 & +0.25 & 0.1738 & 0.7551 & -0.0041 \\
\hline 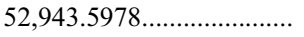 & 2003.8292 & -86.20 & +104.52 & +3.30 & +0.56 & +7.89 & -0.38 & 0.3292 & 0.7604 & -0.0037 \\
\hline $52,978.5239 \ldots \ldots \ldots \ldots \ldots \ldots \ldots \ldots$ & 2003.9248 & -95.01 & +106.13 & +2.53 & +0.09 & -1.78 & -1.35 & 0.2127 & 0.7664 & -0.0033 \\
\hline $53,006.4893 \ldots \ldots \ldots \ldots \ldots \ldots \ldots . .$. & 2004.0013 & -74.36 & +79.73 & +4.33 & -0.60 & +1.33 & +0.30 & 0.1300 & 0.7713 & -0.0029 \\
\hline $53,030.4508 \ldots$. & 2004.0669 & -83.70 & +91.83 & +4.00 & -0.09 & +0.00 & -0.16 & 0.3411 & 0.7754 & -0.0026 \\
\hline
\end{tabular}


TABLE 1-Continued

\begin{tabular}{|c|c|c|c|c|c|c|c|c|c|c|}
\hline $\begin{array}{c}\text { HJD } \\
(2,400,000+)\end{array}$ & Year & $\begin{array}{c}\mathrm{RV}_{\mathrm{Aa}} \\
\left(\mathrm{km} \mathrm{s}^{-1}\right)\end{array}$ & $\begin{array}{c}\mathrm{RV}_{\mathrm{Ab}} \\
\left(\mathrm{km} \mathrm{s}^{-1}\right)\end{array}$ & $\begin{array}{c}\mathrm{RV}_{\mathrm{B}} \\
\left(\mathrm{km} \mathrm{s}^{-1}\right)\end{array}$ & $\begin{array}{c}(O-C)_{\mathrm{Aa}} \\
\left(\mathrm{km} \mathrm{s}^{-1}\right)\end{array}$ & $\begin{array}{c}(O-C)_{\mathrm{Ab}} \\
\left(\mathrm{km} \mathrm{s}^{-1}\right)\end{array}$ & $\begin{array}{l}(O-C)_{\mathrm{B}} \\
\left(\mathrm{km} \mathrm{s}^{-1}\right)\end{array}$ & Inner Phase $^{\mathrm{a}}$ & Outer Phase $^{\mathrm{a}}$ & $\begin{array}{c}\Delta T^{\mathrm{b}} \\
\text { (days) }\end{array}$ \\
\hline $53,127.8133 \ldots$ & 2004.3335 & +67.91 & -114.52 & +4.48 & +1.38 & +0.82 & -0.20 & 0.8316 & 0.7922 & -0.0012 \\
\hline $53,181.6702 \ldots \ldots \ldots \ldots \ldots \ldots \ldots$ & 2004.4810 & +75.66 & -131.09 & +3.90 & -0.23 & -2.60 & -1.05 & 0.7824 & 0.8015 & -0.0004 \\
\hline $53,187.6941 \ldots \ldots \ldots \ldots \ldots \ldots \ldots$ & 2004.4975 & -81.15 & +92.63 & +6.52 & +0.20 & +4.74 & +1.54 & 0.3495 & 0.8026 & -0.0003 \\
\hline $53,202.6395 \ldots \ldots \ldots \ldots \ldots \ldots \ldots . .$. & 2004.5384 & +74.82 & -131.70 & +4.91 & -1.12 & -3.04 & -0.15 & 0.7184 & 0.8052 & -0.0001 \\
\hline $53,275.6923 \ldots \ldots \ldots \ldots \ldots \ldots \ldots$ & 2004.7384 & +62.31 & -103.54 & +4.43 & +1.39 & +4.79 & -0.98 & 0.8495 & 0.8178 & +0.0010 \\
\hline $53,304.6418 \ldots \ldots \ldots \ldots \ldots \ldots \ldots$ & 2004.8176 & -93.92 & +88.17 & +5.98 & -2.70 & -12.75 & +0.44 & 0.1862 & 0.8228 & +0.0015 \\
\hline $53,323.6007 \ldots \ldots \ldots \ldots \ldots \ldots \ldots$ & 2004.8695 & -98.17 & +111.57 & +5.96 & -0.36 & +1.66 & +0.34 & 0.2654 & 0.8261 & +0.0018 \\
\hline $53,342.5272 \ldots \ldots \ldots \ldots \ldots \ldots \ldots$ & 2004.9214 & -86.33 & +102.44 & +3.49 & +0.84 & +7.26 & -2.21 & 0.3308 & 0.8294 & +0.0021 \\
\hline 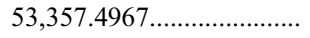 & 2004.9623 & +72.33 & -127.89 & +5.58 & -2.28 & -0.35 & -0.18 & 0.7100 & 0.8319 & +0.0023 \\
\hline $53,372.4574 \ldots \ldots \ldots \ldots \ldots \ldots \ldots$ & 2005.0033 & -54.77 & +49.58 & +4.99 & +0.63 & -1.77 & -0.83 & 0.0855 & 0.8345 & +0.0025 \\
\hline $53,480.8610 \ldots \ldots \ldots \ldots \ldots \ldots \ldots \ldots$ & 2005.3001 & -96.41 & +112.01 & +8.87 & +0.37 & +4.08 & +2.68 & 0.2812 & 0.8533 & +0.0043 \\
\hline
\end{tabular}

a Computed from the ephemeris in the last column of Table 10.

b Corrections for light-travel time (see text).

September and 2003 September, which number 732, 738, 739, and 740 in the $B, V, R$, and $I$ passbands, respectively. The estimated precision of these differential measurements is 0.011 mag in $B, 0.008 \mathrm{mag}$ in $V, 0.009 \mathrm{mag}$ in $R$, and $0.012 \mathrm{mag}$ in $I$. The comparison stars used for these two data sets are given in Table 8 . The URSA photometry was referenced to the magnitude corresponding to the sum of the intensities of all four comparison stars in order to improve the measurement accuracy.

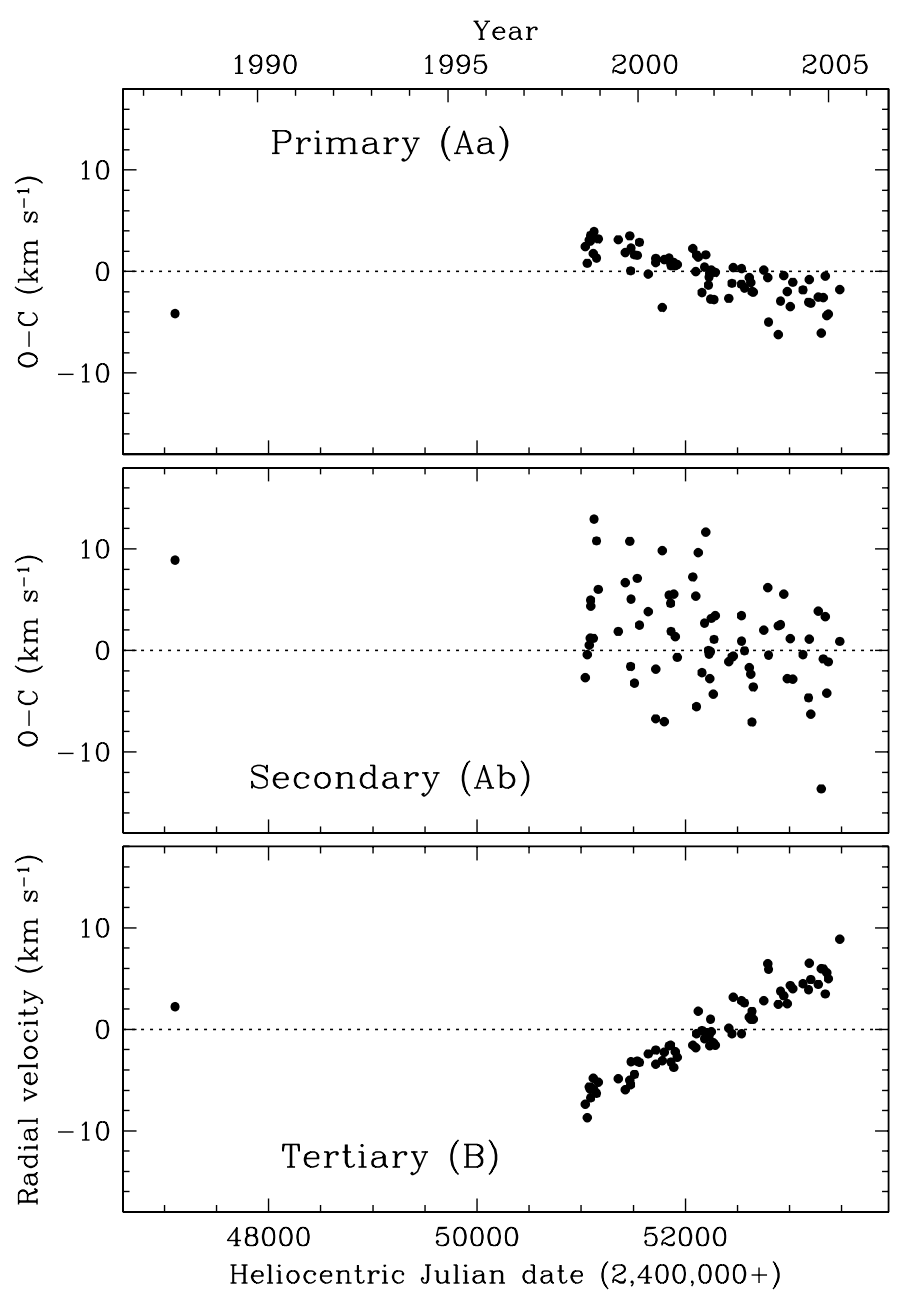

FIG. 2.- Radial velocity residuals for the primary and secondary of V1061 Cyg from a preliminary double-lined orbital solution, and radial velocities for the tertiary. The trends indicate that the system is a hierarchical triple.
Because the eclipse timings for V1061 Cyg described earlier show changes due to motion about the center of mass of the triple system, light elements used in analyzing the photometric data were based on contemporary observations made between 2001 March and 2004 October with the URSA telescope. As seen in Figure 3, the curvature of the $O-C$ residuals is relatively small in this interval, and a linear ephemeris is sufficient for our purposes. A fit to 12 primary minima (see Table 2 ) yielded

$$
\operatorname{Min} \mathrm{I}(\mathrm{HJD})=2,452,015.90562(7)+2.34663383(33) E,
$$

where $E$ is the number of cycles elapsed from the epoch of reference and the values in parentheses represent the uncertainties of the elements in units of the last digit. Photometric data were then phased according to these elements.

The URSA and Gettysburg data sets were analyzed with the Nelson-Davis-Etzel (NDE) model (EBOP code) as described by Etzel (1981) and Popper \& Etzel (1981). Although other models may be more sophisticated, this program is perfectly adequate for well-detached systems such as V1061 Cyg. The main adjustable parameters in the NDE model are the relative surface brightness $\left(J \equiv J_{\mathrm{Ab}} / J_{\mathrm{Aa}}\right)$ of the secondary of the eclipsing binary (star $\mathrm{Ab}$ ) in units of that of the primary (Aa), the relative radius of the primary $\left(r_{\mathrm{Aa}}\right)$ in units of the separation, the ratio of radii $(k \equiv$ $\left.r_{\mathrm{Ab}} / r_{\mathrm{Aa}}\right)$, the inclination of the orbit of the binary $\left(i_{\mathrm{A}}\right)$, the limbdarkening coefficients $\left(x_{\mathrm{Aa}}\right.$ and $\left.x_{\mathrm{Ab}}\right)$, the eccentricity parameters $e \cos \omega$ and $e \sin \omega$, and the amount of third light $\left(l_{\mathrm{B}}\right.$, expressed as a fraction of the total light), which is significant in our case. The luminosity due to the reflection effect was computed from bolometric theory. The mass ratio $(q=0.7266)$ was adopted from the spectroscopic results in $\S 3$. Tests showed that the orbital eccentricity was probably not significantly different from zero, consistent with the results described later, so $e$ was fixed at zero for the photometric modeling. For the URSA data the EBOP algorithm converged with all variables free, except for the gravity-brightening coefficients $y$, which were set from theory (Alencar \& Vaz 1997; Claret 1998). For the Gettysburg data sets the limb-darkening coefficients $x$ had to be fixed at theoretical values (Al-Naimiy 1978; Wade \& Rucinski 1985; Díaz-Cordovés et al. 1995), as were the gravity-brightening coefficients. In addition, for the Gettysburg data the values of third light in the spectral bands $B, R$, and $I$ had to be estimated from the temperature of the third star based on the spectroscopic analysis in $\S 2.1$. The third light value in $V$ was adopted from the URSA data solution $\left(l_{\mathrm{B}}=0.144\right)$. For these reasons, the final mean geometric parameters based on the Gettysburg 
TABLE 2

Measured Times of Eclipse for V1061 Cyg

\begin{tabular}{|c|c|c|c|c|c|c|c|}
\hline $\begin{array}{c}\text { HJD } \\
(2,400,000+)\end{array}$ & $\begin{array}{c}\sigma \\
\text { (days) }\end{array}$ & Year & Type $^{\mathrm{a}}$ & Eclipse $^{\mathrm{b}}$ & $E$ & $\begin{array}{l}O-C \\
\text { (days) }\end{array}$ & References \\
\hline $26,355.245 \ldots \ldots \ldots \ldots \ldots \ldots \ldots \ldots \ldots$ & 0.021 & 1931.0342 & pg & 1 & -10935.0 & +0.02093 & 1 \\
\hline $26,512.460 \ldots \ldots \ldots \ldots \ldots \ldots \ldots \ldots \ldots$ & 0.021 & 1931.4646 & $\mathrm{pg}$ & 1 & -10868.0 & +0.00762 & 1 \\
\hline $26,925.476 \ldots \ldots \ldots \ldots \ldots \ldots \ldots \ldots \ldots \ldots \ldots \ldots \ldots$ & 0.021 & 1932.5954 & pg & 1 & -10692.0 & +0.00714 & 1 \\
\hline $26,958.310 \ldots \ldots \ldots$ & 0.021 & 1932.6853 & pg & 1 & -10678.0 & -0.01237 & 1 \\
\hline $26,958.335 \ldots \ldots \ldots$ & 0.021 & 1932.6854 & pg & 1 & -10678.0 & +0.01263 & 1 \\
\hline $27,183.594 \ldots \ldots \ldots \ldots \ldots \ldots \ldots \ldots \ldots . .$. & 0.021 & 1933.3021 & pg & 1 & -10582.0 & -0.00930 & 1 \\
\hline $28,692.483 \ldots \ldots \ldots \ldots \ldots \ldots \ldots \ldots \ldots . .$. & 0.021 & 1937.4332 & pg & 1 & -9939.0 & -0.02197 & 1 \\
\hline $28,753.487 \ldots \ldots \ldots \ldots \ldots \ldots \ldots \ldots$ & 0.021 & 1937.6002 & pg & 1 & -9913.0 & -0.03072 & 1 \\
\hline $36,788.465 \ldots \ldots \ldots \ldots \ldots \ldots \ldots \ldots$ & 0.021 & 1959.5988 & pg & 1 & -6489.0 & +0.02863 & 1 \\
\hline $36,868.261 \ldots \ldots \ldots \ldots \ldots \ldots \ldots \ldots$ & 0.021 & 1959.8173 & pg & 1 & -6455.0 & +0.03879 & 1 \\
\hline $44,166.347 \ldots \ldots \ldots \ldots \ldots \ldots \ldots \ldots \ldots$ & 0.011 & 1979.7983 & $\mathrm{v}$ & 1 & -3345.0 & +0.00712 & 2 \\
\hline $45,229.383 \ldots \ldots \ldots \ldots \ldots \ldots \ldots \ldots \ldots$ & 0.011 & 1982.7088 & $\mathrm{v}$ & 1 & -2892.0 & +0.00127 & 2 \\
\hline 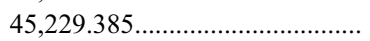 & 0.021 & 1982.7088 & $\mathrm{pg}$ & 1 & -2892.0 & +0.00327 & 2 \\
\hline $45,933.377 \ldots \ldots \ldots \ldots \ldots \ldots \ldots$ & 0.011 & 1984.6362 & $\mathrm{v}$ & 1 & -2592.0 & -0.00071 & 2 \\
\hline $46,001.438 \ldots \ldots \ldots \ldots \ldots \ldots \ldots$ & 0.011 & 1984.8226 & $\mathrm{v}$ & 1 & -2563.0 & +0.00756 & 2 \\
\hline $46,651.4515 \ldots \ldots \ldots \ldots \ldots \ldots \ldots \ldots$ & 0.0015 & 1986.6022 & pe & 1 & -2286.0 & +0.00191 & 3 \\
\hline $46,705.420 \ldots \ldots \ldots \ldots \ldots \ldots \ldots \ldots \ldots$ & 0.011 & 1986.7500 & $\mathrm{v}$ & 1 & -2263.0 & -0.00215 & 2 \\
\hline 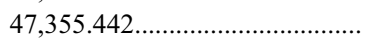 & 0.011 & 1988.5296 & $\mathrm{v}$ & 1 & -1986.0 & +0.00431 & 2 \\
\hline $47,362.476 \ldots \ldots \ldots \ldots \ldots \ldots \ldots \ldots \ldots$ & 0.0015 & 1988.5489 & pe & 1 & -1983.0 & -0.00095 & 2 \\
\hline $48,972.2716 \ldots \ldots \ldots \ldots \ldots \ldots \ldots \ldots$ & 0.0007 & 1992.9563 & pe & 1 & -1297.0 & -0.00127 & 2 \\
\hline $48,972.2735 \ldots \ldots \ldots \ldots \ldots \ldots \ldots \ldots$ & 0.0005 & 1992.9563 & pe & 1 & -1297.0 & +0.00063 & 2 \\
\hline $49,528.413 \ldots \ldots \ldots \ldots \ldots \ldots \ldots \ldots$ & 0.011 & 1994.4789 & $\mathrm{v}$ & 1 & -1060.0 & -0.02707 & 2 \\
\hline $49,535.4799 \ldots \ldots \ldots \ldots \ldots \ldots \ldots \ldots \ldots \ldots$ & 0.0005 & 1994.4982 & pe & 1 & -1057.0 & -0.00024 & 4 \\
\hline $49,535.4802 \ldots \ldots \ldots \ldots \ldots \ldots \ldots \ldots$ & 0.0008 & 1994.4982 & pe & 1 & -1057.0 & +0.00006 & 4 \\
\hline $49,941.4566 \ldots \ldots \ldots \ldots \ldots \ldots \ldots \ldots$ & 0.0003 & 1995.6097 & pe & 1 & -884.0 & -0.00046 & 5 \\
\hline $49,941.4572 \ldots \ldots \ldots \ldots \ldots \ldots \ldots$ & 0.0003 & 1995.6097 & pe & 1 & -884.0 & +0.00014 & 5 \\
\hline $50,286.4260 \ldots \ldots \ldots \ldots \ldots \ldots \ldots \ldots \ldots \ldots \ldots$ & 0.011 & 1996.5542 & $\mathrm{v}$ & 1 & -737.0 & +0.00719 & 2 \\
\hline $51,159.3771 \ldots \ldots \ldots \ldots \ldots \ldots \ldots \ldots \ldots$ & 0.0022 & 1998.9442 & pe & 1 & -365.0 & -0.00136 & 2 \\
\hline $51,159.3787 \ldots \ldots \ldots \ldots \ldots \ldots \ldots \ldots$ & 0.0008 & 1998.9442 & pe & 1 & -365.0 & +0.00024 & 2 \\
\hline $51,159.3789 \ldots \ldots \ldots \ldots \ldots \ldots \ldots \ldots$ & 0.0009 & 1998.9442 & pe & 1 & -365.0 & +0.00044 & 6 \\
\hline $51,159.3791 \ldots \ldots \ldots \ldots \ldots \ldots \ldots \ldots \ldots$ & 0.0007 & 1998.9442 & pe & 1 & -365.0 & +0.00064 & 2 \\
\hline $52,015.90554 \ldots \ldots \ldots \ldots \ldots \ldots \ldots \ldots \ldots \ldots \ldots \ldots \ldots$ & 0.00010 & 2001.2893 & pe & 1 & 0.0 & +0.00013 & 7 \\
\hline $52,095.6909 \ldots \ldots \ldots \ldots \ldots \ldots \ldots \ldots \ldots \ldots$ & 0.0002 & 2001.5077 & pe & 1 & +34.0 & -0.00029 & 8 \\
\hline $52,102.73118 \ldots \ldots \ldots \ldots \ldots \ldots \ldots \ldots \ldots$ & 0.00013 & 2001.5270 & pe & 1 & +37.0 & +0.00008 & 8 \\
\hline $52,109.77080 \ldots \ldots \ldots \ldots \ldots \ldots \ldots \ldots$ & 0.00012 & 2001.5463 & pe & 1 & +40.0 & -0.00022 & 8 \\
\hline $52,149.6636 \ldots \ldots \ldots \ldots \ldots \ldots \ldots \ldots \ldots \ldots$ & 0.0003 & 2001.6555 & pe & 1 & +57.0 & -0.00029 & 8 \\
\hline $52,448.8603 \ldots \ldots \ldots \ldots \ldots \ldots \ldots \ldots \ldots \ldots \ldots \ldots \ldots \ldots \ldots$ & 0.0011 & 2002.4746 & pe & 2 & +184.5 & +0.00031 & 9 \\
\hline $52,482.8861 \ldots \ldots \ldots \ldots \ldots \ldots \ldots \ldots \ldots \ldots \ldots \ldots \ldots$ & 0.0003 & 2002.5678 & pe & 1 & +199.0 & -0.00006 & 9 \\
\hline $52,589.6558 \ldots \ldots \ldots \ldots \ldots \ldots \ldots \ldots \ldots \ldots \ldots \ldots$ & 0.0018 & 2002.8601 & pe & 2 & +244.5 & -0.00210 & 9 \\
\hline $52,602.56431 \ldots \ldots \ldots \ldots \ldots \ldots \ldots \ldots \ldots \ldots$ & 0.00010 & 2002.8955 & pe & 1 & +250.0 & -0.00006 & 9 \\
\hline $52,609.60438 \ldots \ldots \ldots \ldots \ldots \ldots \ldots \ldots \ldots \ldots \ldots \ldots$ & 0.00010 & 2002.9147 & pe & 1 & +253.0 & +0.00012 & 9 \\
\hline $52,786.7736 \ldots \ldots \ldots \ldots \ldots \ldots \ldots \ldots \ldots \ldots \ldots \ldots$ & 0.0014 & 2003.3998 & pe & 2 & +328.5 & -0.00113 & 10 \\
\hline 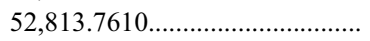 & 0.0002 & 2003.4737 & pe & 1 & +340.0 & +0.00007 & 10 \\
\hline $52,834.8804 \ldots \ldots \ldots \ldots \ldots \ldots \ldots \ldots \ldots \ldots \ldots \ldots$ & 0.0003 & 2003.5315 & pe & 1 & +349.0 & -0.00017 & 10 \\
\hline $52,867.7336 \ldots \ldots \ldots \ldots \ldots \ldots \ldots \ldots \ldots \ldots$ & 0.0003 & 2003.6214 & pe & 1 & +363.0 & +0.00027 & 10 \\
\hline $52,887.6813 \ldots \ldots \ldots \ldots \ldots \ldots \ldots \ldots \ldots \ldots \ldots \ldots$ & 0.0014 & 2003.6761 & pe & 2 & +371.5 & +0.00165 & 10 \\
\hline $52,907.62597 \ldots \ldots \ldots \ldots \ldots \ldots \ldots \ldots$ & 0.00012 & 2003.7307 & pe & 1 & +380.0 & +0.00001 & 10 \\
\hline $53,145.8083 \ldots \ldots \ldots \ldots \ldots \ldots \ldots \ldots \ldots \ldots \ldots \ldots \ldots$ & 0.0022 & 2004.3828 & pe & 2 & +481.5 & +0.00014 & 11 \\
\hline 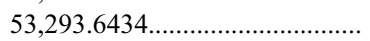 & 0.0011 & 2004.7875 & pe & 2 & +544.5 & -0.00178 & 11 \\
\hline
\end{tabular}

Notes.-Timing uncertainties $(\sigma)$ have been determined or adjusted as described in the text. $O-C$ residuals are from the global solution in $\S 3$.

${ }^{a}$ Technique: $\mathrm{pg}=$ photographic; $\mathrm{v}=$ visual; $\mathrm{pe}=$ photoelectric/CCD.

b 1 = primary; 2 = secondary.

References.-(1) Strohmeier et al. 1962; (2) Kreiner et al. 2001; (3) Diethelm 1986; (4) Agerer \& Hübscher 1995; (5) Agerer \& Hübscher 1996; (6) Ogłoza et al. 2000; (7) Lacy 2001; (8) Lacy et al. 2002; (9) Lacy 2002; (10) Lacy 2003; (11) Lacy 2004.

data have been assigned uncertainties 2 times the internal estimates, consistent with our experience with EBOP in these situations.

Initial light-curve solutions with the URSA data showed that observations during the night of JD 2,452,054 (2001 May 24), which followed a secondary eclipse, were significantly fainter than the fitted orbit by $\sim 0.025 \mathrm{mag}$. No other observations at this phase (0.57) were collected until the 2003-2004 seasons. In addition, data on JD 2,453,218 and JD 2,453,232 (2004 season) obtained during and after secondary eclipse were seen to be brighter than the fitted curve by $\sim 0.01 \mathrm{mag}$. Measurements taken 12 days earlier at a similar phase appear to be normal, as are those taken 2 months later. Possible explanations for these deviations include some unknown calibration problem in the data 


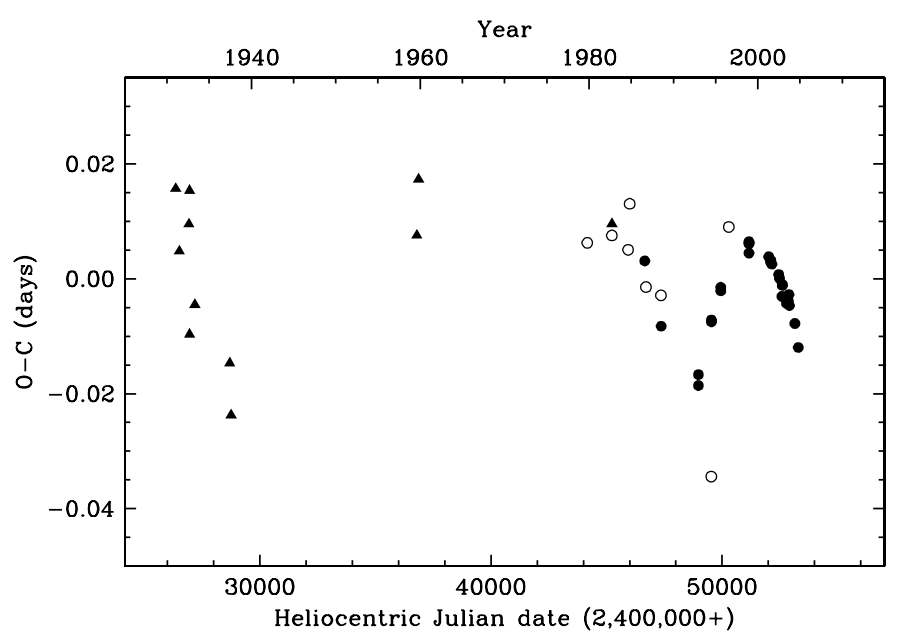

FIG. 3. $-O-C$ diagram of all available times of eclipse for V1061 Cyg, from a preliminary linear ephemeris derived from a fit to the photoelectric ( filled circles), visual (open circles), and photographic measurements (triangles) for both the primary and secondary minima. Oscillations suggesting a third body are obvious, particularly in the recent data.

reductions, or transitory spots on one or both stars that change on timescales of a month or two. The latter would not be unusual given the strong X-ray emission from the system (Voges et al. 1999), indicative of chromospheric activity (see $\S 5.2$ ). If due to spots, the extent of the active regions appears to be quite large (up to $40^{\circ}-50^{\circ}$ in longitude), judging from the phase interval affected. Tests showed that removal of these URSA observations had an insignificant effect on the results other than decreasing the scatter. We have thus chosen to exclude the data on those three nights from the final fitting process. Further support for intrinsic variations is given by similar deviations seen in the Gettysburg data at other phases, particularly phase $\sim 0.45$, as shown below in Figure 7. The angular extent of this particular feature is again $\sim 40^{\circ}$. A hint of a dimming at about the same phase may be present also in the URSA observations during the same season, although the data sampling is not optimal for confirming this.

The results of our photometric analysis are given in Table 9, separately for each of the Gettysburg passbands and for URSA $V$. The mean of the Gettysburg results is also listed, with uncertainties that account for the scatter between the $B V R I$ results. There is excellent agreement in the geometric elements between the two data sets. The light curve and residuals for the URSA measurements are shown in Figure 4, and enlargements of the regions around the minima are shown in Figures 5 and 6. The Gettysburg light curves and residuals are shown in Figure 7 . The binary is well detached: the stars occupy only $\sim 7 \%$ and $\sim 2 \%$ by volume

TABLE 3

Differential $V$-Band Photometry for V1061 Cyg FROM THE URSA TELESCOPE

\begin{tabular}{|c|c|}
\hline $\begin{array}{c}\text { HJD } \\
(2,400,000+)\end{array}$ & $\begin{array}{c}\Delta V \\
(\mathrm{mag})\end{array}$ \\
\hline $52,102.73110 \ldots$ & -0.101 \\
\hline $52,428.91322 .$. & -0.091 \\
\hline $52,095.69134 \ldots$ & -0.091 \\
\hline $52,076.91832 \ldots$ & -0.083 \\
\hline 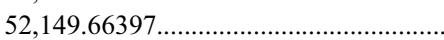 & -0.102 \\
\hline
\end{tabular}

Notes.-Table 3 is published in its entirety in the electronic edition of the Astrophysical Journal. A portion is shown here for guidance regarding its form and content.
TABLE 4

Differential $B$-Band Photometry for V1061 Cyg from Gettysburg

HJD
$(2,400,000+)$$\quad \begin{gathered}\Delta B \\ (\mathrm{mag})\end{gathered}$

Notes.-Table 4 is published in its entirety in the electronic edition of the Astrophysical Journal. A portion is shown here for guidance regarding its form and content.

of their critical equipotential surfaces. Both components are nearly spherical in shape (oblateness $<0.005$, as defined by Etzel 1981). The secondary eclipse is total, and the fraction of the primary light blocked at phase 0.0 is $40 \%$.

A common problem in solving the light curves of some eclipsing systems is the indeterminacy in the ratio of the radii, particularly when the components are similar and the eclipses partial. As a test, we fitted the URSA light curves with fixed values of $k$ over a wide range around the best-fit value of 0.603 . Solutions converged only between 0.55 and 0.63 . Some parameters such as $l_{\mathrm{B}}$, and to a lesser extent the relative brightness of the secondary $\left(l_{\mathrm{Ab}}\right)$, showed a very strong sensitivity to $k$. Only fits in the vicinity of $k=0.603$ (which minimizes the sum of residuals squared) resulted in values of $l_{\mathrm{B}}$ and $l_{\mathrm{Ab}}$ consistent with the spectroscopic estimates from $\S 2.1$ (after a small correction from the mean wavelength of $5187 \AA$ to the $V$ band). We take this as an indication that the adopted fits are realistic.

\subsection{Astrometry}

V1061 Cyg was observed by the Hipparcos satellite under the designation HIP 104263, from 1989 December through 1993 February. The measured trigonometric parallax is $\pi_{\text {Hip }}=6.25 \pm$ 1.06 mas. The photometric variability of the star with the known 2.35 day period was clearly detected in the more than 120 brightness measurements made over the duration of the mission. In addition, even though the wide orbit in V1061 Cyg is much too small to be spatially resolved by Hipparcos, the astrometric solution revealed perturbations in the motion across the sky that led to its classification as a "variability-induced mover" (VIM). These perturbations arise from the fact that the center of light of the triple system shifts during the eclipses due to the change in the relative brightness between the binary and the third star. The shift is approximately 25 mas, which the satellite was able to detect. As a first-order approximation the Hipparcos team assumed

TABLE 5

Differential $V$-Band Photometry for V1061 Cyg from Gettysburg

HJD
$(2,400,000+)$$\quad \begin{gathered}\Delta V \\ (\mathrm{mag})\end{gathered}$

Notes.-Table 5 is published in its entirety in the electronic edition of the Astrophysical Journal. A portion is shown here for guidance regarding its form and content. 
TABLE 6

Differential $R$-Band Photometry for V1061 Cyg from Gettysburg

HJD
$(2,400,000+)$$\quad \begin{gathered}\Delta R \\ (\mathrm{mag})\end{gathered}$

Notes.-Table 6 is published in its entirety in the electronic edition of the Astrophysical Journal. A portion is shown here for guidance regarding its form and content.

that the angular separation between the binary and the third star was constant, so that the motion of the photocenter is along a straight line connecting them. With this they were able to infer a lower limit to the separation of approximately 70 mas and a position angle (north through east) for the third star relative to the binary of $319^{\circ} \pm 14^{\circ}$. This linear and periodic motion of the photocenter was accounted for in deriving the position, proper motion, and parallax of V1061 Cyg, as reported in the catalog.

In reality the third star and the binary are in orbit around each other, and from Figure 3 the period appears to be of the order of $15 \mathrm{yr}$. The $3.2 \mathrm{yr}$ interval of the Hipparcos observations represents a nonnegligible fraction of a cycle, and depending on the orientation of the orbit, this may have an impact on the derived parallax and proper motion, as well as the inferred motion of the photocenter. In fact, turning the problem around, the Hipparcos observations can potentially provide valuable constraints on the outer orbit. Thus, we incorporate these observations (in the form of the intermediate astrometric data, or "abscissa residuals") into the orbital solution described next.

\section{ORBITAL SOLUTION}

Due to the much larger orbit of the third star compared to the eclipsing binary in V1061 Cyg, to first order we assume here that the hierarchical triple system may be separated into an inner orbit and an outer orbit, the latter treated as a "binary" composed of the third star (B) and the center of mass of the eclipsing pair (A). Orbital elements that refer to the outer orbit are indicated below with the subindex "AB," and those pertaining to the inner orbit are distinguished with a subindex "A."

In addition to providing the spectroscopic elements of the inner orbit, in principle the radial velocity measurements yield also the elements of the outer orbit, except that in our case the coverage is insufficient for that purpose (see Fig. 2), and the period of the outer orbit $\left(P_{\mathrm{AB}}\right)$ is undetermined. Furthermore, the semi-

TABLE 7

Differential $I$-Band Photometry for V1061 Cyg from Gettysburg

\begin{tabular}{|c|c|}
\hline $\begin{array}{c}\text { HJD } \\
(2,400,000+)\end{array}$ & $\begin{array}{c}\Delta I \\
(\mathrm{mag})\end{array}$ \\
\hline $52,548.59172 .$. & 0.191 \\
\hline $52,541.55240 \ldots$ & 0.187 \\
\hline $52,548.59402 \ldots$ & 0.190 \\
\hline $52,541.55460 \ldots$ & 0.172 \\
\hline 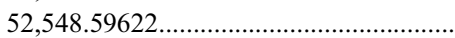 & 0.185 \\
\hline
\end{tabular}

Notes.-Table 7 is published in its entirety in the electronic edition of the Astrophysical Journal. A portion is shown here for guidance regarding its form and content.
TABLE 8

Comparison Stars for V1061 Cyg

\begin{tabular}{|c|c|c|c|c|}
\hline Star & $\begin{array}{c}\text { R.A. } \\
(\text { J2000.0) }\end{array}$ & $\begin{array}{c}\text { Decl. } \\
(\mathrm{J} 2000.0)\end{array}$ & $B_{T}$ & $V_{T}$ \\
\hline \multicolumn{5}{|c|}{ URSA } \\
\hline GSC $03600-00278 \ldots \ldots \ldots$ & 210734.082 & +520651.60 & 11.210 & 10.605 \\
\hline GSC $03600-00377 \ldots \ldots . .$. & 210722.127 & +520137.07 & 12.555 & 11.824 \\
\hline GSC $03600-00423 \ldots \ldots \ldots$. & 210655.420 & +520249.14 & 11.837 & 11.456 \\
\hline GSC $03600-00443 \ldots \ldots \ldots$ & 210640.440 & +520802.96 & 13.087 & 11.451 \\
\hline \multicolumn{5}{|c|}{ Gettysburg } \\
\hline GSC $03600-00425 \ldots \ldots \ldots$ & 210649.626 & +520017.08 & 9.261 & 9.026 \\
\hline GSC $03600-00259 \ldots \ldots \ldots$ & 210740.472 & +515256.94 & 11.610 & 10.318 \\
\hline
\end{tabular}

Note.-Coordinates and magnitudes $B_{T}$ and $V_{T}$ are taken from the Tycho-2 catalog (Høg et al. 2000).

amplitudes of the velocity variation in the wide orbit $\left(K_{\mathrm{A}}\right.$ and $\left.K_{\mathrm{B}}\right)$ are poorly constrained because the observations do not cover the velocity extrema. The times of eclipse help in two important ways: they constrain the period $(\sim 15 \mathrm{yr}$ from a preliminary analysis), and they constrain the amplitude of the third-body effect $\left(K_{O-C}\right)$, which is directly related to $K_{\mathrm{A}}$ through $K_{O-C}=K_{\mathrm{A}}(1-$ $\left.e_{\mathrm{AB}}^{2}\right)^{1 / 2} / 2 \pi c$, where $e_{\mathrm{AB}}$ is the eccentricity of the outer orbit and $c$ is the speed of light. The fact that the inner binary is eclipsing allows the masses of the three stars to be determined. For this we incorporate the angle $i_{\mathrm{A}}$ from $\S 2.3$ into the solution, along with its uncertainty.

The use of the Hipparcos data introduces several other orbital elements into the problem that are constrained by the astrometry, including the inclination angle of the wide orbit $\left(i_{\mathrm{AB}}\right)$ and the position angle of the ascending node $\left(\Omega_{\mathrm{AB}}\right.$, referred to the equinox of J2000.0). In addition, we must solve for corrections to the catalog values of the position of the barycenter $\left(\Delta \alpha^{*}, \Delta \delta\right)$ at the mean catalog epoch of 1991.25 , corrections to the proper motions $\left(\Delta \mu_{\alpha}^{*}, \Delta \mu_{\delta}\right)$, and corrections to the trigonometric parallax $(\Delta \pi) .{ }^{7}$ Because the inner binary is eclipsing, there is redundancy in that the angle $i_{\mathrm{AB}}$ can also be derived purely from the spectroscopic elements and the known value of $i_{\mathrm{A}}$. We may thus eliminate one parameter, and for practical reasons we have chosen to eliminate $K_{\mathrm{B}}$ and to retain $i_{\mathrm{AB}}$ explicitly as an adjustable variable.

Altogether there are 19 unknowns in the least-squares problem: $\left\{P_{\mathrm{A}}, K_{\mathrm{Aa}}, K_{\mathrm{Ab}}, e_{\mathrm{A}}, \omega_{\mathrm{Aa}}, T_{\mathrm{A}}\right\}$ for the inner orbit, $\left\{P_{\mathrm{AB}}, K_{\mathrm{A}}, i_{\mathrm{AB}}, e_{\mathrm{AB}}\right.$, $\left.\omega_{\mathrm{A}}, \Omega_{\mathrm{AB}}, T_{\mathrm{AB}}\right\}$ for the outer orbit, the center-of-mass velocity $\gamma$, and the Hipparcos-related elements $\left\{\Delta \alpha^{*}, \Delta \delta, \Delta \mu_{\alpha}^{*}, \Delta \mu_{\delta}, \Delta \pi\right\}$. The longitudes of periastron $\omega$ refer to the star or system indicated; $T_{\mathrm{A}}$ is the time of primary eclipse in the inner orbit, and $T_{\mathrm{AB}}$ represents the time of periastron passage in the outer orbit.

The amplitude of the motion of the photocenter in angular units, $\alpha_{\mathrm{ph}}^{\prime \prime}$, is a function of the fractional mass and luminosity of the third star relative to the system as a whole. It is given by $\alpha_{\mathrm{ph}}^{\prime \prime}=a_{\mathrm{AB}}^{\prime \prime}(B-\beta)$, in which the mass fraction is $B \equiv M_{\mathrm{B}} /\left(M_{\mathrm{Aa}}+\right.$ $\left.M_{\mathrm{Ab}}+M_{\mathrm{B}}\right)$ and the luminosity fraction is $\beta \equiv L_{\mathrm{B}} /\left(L_{\mathrm{Aa}}+L_{\mathrm{Ab}}+\right.$ $\left.L_{\mathrm{B}}\right)$. With the definition of third light used in EBOP, $\beta \equiv l_{\mathrm{B}}$. The symbol $a_{\mathrm{AB}}^{\prime \prime}$ represents the relative semimajor axis of the wide orbit expressed in angular measure. The notation $a^{\prime \prime}$ is used here to distinguish from the semimajor axis in linear units, $a$. The mass fraction $B$ can be expressed in terms of other elements, and $a_{\mathrm{AB}}^{\prime \prime}$ follows from knowledge of $P_{\mathrm{AB}}$, the masses, the parallax, and the

\footnotetext{
${ }^{7}$ Following the practice in the Hipparcos catalog, we define $\Delta \alpha^{*} \equiv \Delta \alpha \cos \delta$ and $\Delta \mu_{\alpha}^{*} \equiv \Delta \mu_{\alpha} \cos \delta$.
} 
TABLE 9

Light-Curve Solutions for V1061 Cyg

\begin{tabular}{|c|c|c|c|c|c|c|}
\hline \multirow[b]{2}{*}{ Parameter $^{\mathrm{a}}$} & \multicolumn{5}{|c|}{ GetTysbuRG } & \multirow{2}{*}{$\begin{array}{c}\text { URSA } \\
V\end{array}$} \\
\hline & $B$ & V & $R$ & $I$ & Mean & \\
\hline \multirow[t]{2}{*}{$J \equiv J_{\mathrm{Ab}} / J_{\mathrm{Aa}} \ldots \ldots \ldots \ldots \ldots \ldots \ldots$} & 0.398 & 0.472 & 0.525 & 0.590 & $\ldots$ & 0.436 \\
\hline & 0.005 & 0.005 & 0.006 & 0.005 & $\ldots$ & 0.002 \\
\hline \multirow{2}{*}{$r_{\mathrm{Aa}} \ldots \ldots \ldots \ldots \ldots \ldots \ldots \ldots \ldots \ldots \ldots \ldots}$. & 0.1635 & 0.1625 & 0.1635 & 0.1648 & 0.164 & 0.1674 \\
\hline & 0.0009 & 0.0010 & 0.0010 & 0.0009 & 0.002 & 0.0009 \\
\hline \multirow[t]{2}{*}{$r_{\mathrm{Ab}}$} & 0.0959 & 0.0970 & 0.0971 & 0.0994 & 0.097 & 0.1009 \\
\hline & 0.0007 & 0.0008 & 0.0008 & 0.0007 & 0.002 & 0.0006 \\
\hline \multirow[t]{2}{*}{$k \equiv r_{\mathrm{Ab}} / r_{\mathrm{Aa}} \ldots \ldots \ldots \ldots \ldots \ldots \ldots$} & 0.587 & 0.597 & 0.594 & 0.603 & 0.595 & 0.603 \\
\hline & 0.003 & 0.003 & 0.003 & 0.002 & 0.007 & 0.009 \\
\hline \multirow[t]{2}{*}{$i_{\mathrm{A}}(\operatorname{deg})$} & 89.1 & 88.5 & 88.5 & 88.3 & 88.6 & 87.9 \\
\hline & 0.5 & 0.3 & 0.3 & 0.3 & 0.4 & 0.3 \\
\hline \multirow{2}{*}{ 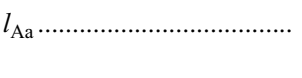 } & 0.763 & 0.737 & 0.718 & 0.699 & $\ldots$ & 0.743 \\
\hline & 0.009 & 0.010 & 0.009 & 0.008 & $\ldots$ & 0.016 \\
\hline \multirow[t]{2}{*}{$l_{\mathrm{Ab}}$} & 0.100 & 0.119 & 0.130 & 0.145 & $\ldots$ & 0.113 \\
\hline & 0.009 & 0.010 & 0.009 & 0.008 & $\ldots$ & 0.016 \\
\hline \multirow{2}{*}{$l_{\mathrm{B}}$} & 0.137 & 0.144 & 0.152 & 0.156 & $\ldots$ & 0.144 \\
\hline & Fixed & Fixed & Fixed & Fixed & $\ldots$ & 0.018 \\
\hline \multirow{2}{*}{ 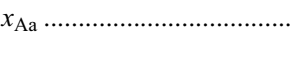 } & 0.74 & 0.50 & 0.48 & 0.35 & $\ldots$ & 0.50 \\
\hline & Fixed & Fixed & Fixed & Fixed & $\ldots$ & 0.03 \\
\hline \multirow[t]{2}{*}{$x_{\mathrm{Ab}} \ldots \ldots \ldots \ldots \ldots \ldots \ldots \ldots \ldots \ldots \ldots$} & 0.83 & 0.60 & 0.54 & 0.42 & $\ldots$ & 0.60 \\
\hline & Fixed & Fixed & Fixed & Fixed & $\ldots$ & 0.03 \\
\hline \multirow[t]{2}{*}{$y_{\mathrm{Aa}} \ldots \ldots \ldots \ldots \ldots \ldots \ldots \ldots \ldots \ldots \ldots \ldots$} & 0.33 & 0.33 & 0.33 & 0.33 & $\ldots$ & 0.33 \\
\hline & Fixed & Fixed & Fixed & Fixed & $\ldots$ & Fixed \\
\hline \multirow[t]{2}{*}{ 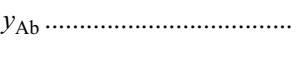 } & 0.41 & 0.41 & 0.41 & 0.41 & $\ldots$ & 0.41 \\
\hline & Fixed & Fixed & Fixed & Fixed & $\ldots$ & Fixed \\
\hline$\sigma(\mathrm{mag})$ & 0.0071 & 0.0082 & 0.0081 & 0.0071 & $\ldots$ & 0.0098 \\
\hline$N_{\text {obs }}$ & 732 & 738 & 739 & 740 & $\ldots$ & 6129 \\
\hline
\end{tabular}

${ }^{a}$ The second row for each parameter indicates the uncertainty (standard deviation), or whether the parameter was held fixed in the fit. All solutions adopt a mass ratio $q=0.7266$ from Table 10 .

application of Kepler's third law. The luminosity fraction $l_{\mathrm{B}}$ needs to be specified in the Hipparcos passband $\left(H_{p}\right)$, which is slightly different from Johnson $V$. To derive it, we have made use of the Hipparcos epoch photometry for V1061 Cyg and solved the light curve with EBOP. Since the measurements available are fewer in number compared to our other light curves, we fixed the geometric

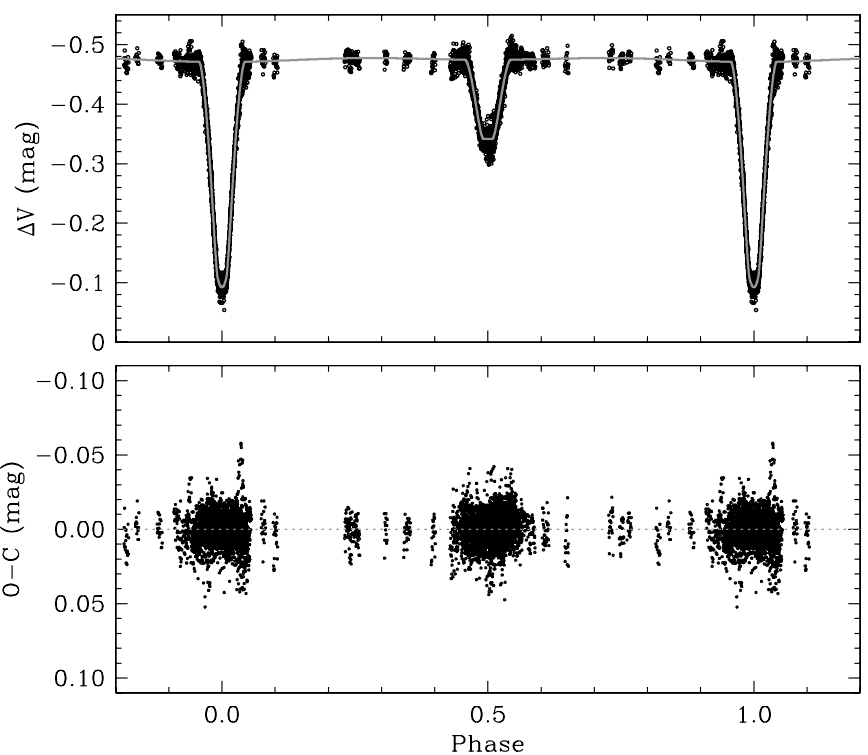

FIG. 4.-URSA $V$-band photometry for V1061 Cyg along with our fitted light curve, and residuals from that fit. [See the electronic edition of the Journal for a color version of this figure.] elements to the average of the URSA and Gettysburg results from Table 9 and solved only for the passband-dependent elements $J$ and $l_{\mathrm{B}}$. Limb-darkening coefficients have little effect and were fixed to their values for the $V$ band. The fit to the Hipparcos photometry is shown in Figure 8. The results we obtained from

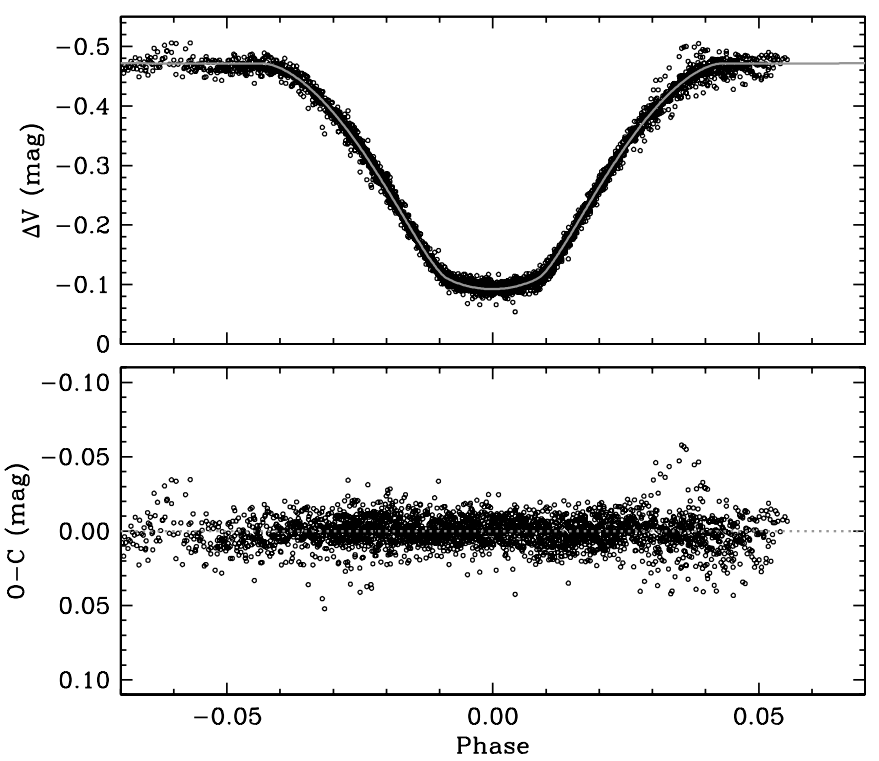

FIG. 5.-URSA V-band photometry for V1061 Cyg around the primary minimum, along with our model light curve. Residuals from the fit are shown in the bottom panel. [See the electronic edition of the Journal for a color version of this figure.] 

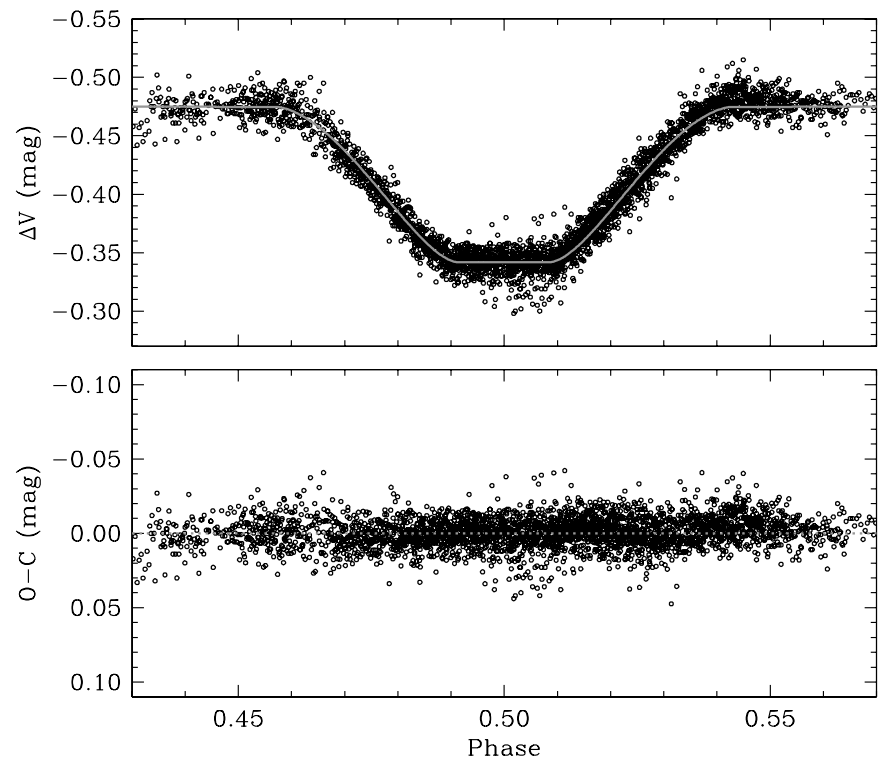

FIG. 6.-URSA V-band photometry for V1061 Cyg around the secondary minimum, along with our model light curve. Residuals from the fit are shown in the bottom panel. Note that the vertical scale in the top panel is different from Fig. 5. [See the electronic edition of the Journal for a color version of this figure.]

this adjustment are $l_{\mathrm{B}}=0.12 \pm 0.02$ and $J=0.44 \pm 0.04\left(H_{p}\right)$. With this value of $l_{\mathrm{B}}$ the photocentric semimajor axis $\alpha_{\mathrm{ph}}^{\prime \prime}$ is completely determined at each iteration of the least-squares problem and is not an additional free parameter in the orbital solution. The formalism for incorporating the abscissa residuals from Hipparcos into the fit follows closely that described by van Leeuwen \& Evans (1998) and Pourbaix \& Jorissen (2000), including the correlations between measurements from the two independent data reduction consortia (ESA 1997).

While the Hipparcos observations provide information that is complementary to that from other available measurements, the astrometric constraint on some of the orbital elements is not particularly strong due to the limited precision of those measurements. Typical uncertainties for a single abscissa residual range from 2 to 4 mas, which is only a few times smaller than the motions we are trying to model. Additional constraints may be placed on the parallax by using the physical properties of stars $\mathrm{Aa}$ and $\mathrm{Ab}$ derived in $\S 4$, which allow the absolute luminosity of each component to be determined very accurately from their effective temperature and radius. This is the same principle that has been used to great advantage for applications such as establishing the distance of external galaxies from eclipsing binaries (e.g., Guinan et al. 1998; Fitzpatrick et al. 2003). From the radii and temperatures in $\S 4$, along with the total apparent magnitude of V1061 Cyg and the fractional luminosities of the components, we obtain parallax estimates of $5.90 \pm 0.26$ mas (Aa) and $5.72 \pm 0.48$ mas $(\mathrm{Ab})$, which are consistent with each other. We incorporate these estimates into the global solution as "measurements," along with their uncertainties.

The delay or advance in the times of eclipse caused by the third star is accounted for as described by Irwin (1952). The bestfit time of primary eclipse $\left(T_{\mathrm{A}}\right)$ given below refers to the center of the elliptical orbit of the eclipsing pair around the common center of mass of the triple, as opposed to the barycenter (see Irwin 1952). For consistency, corrections for light-travel time in the inner binary have also been applied to the dates of the radial velocity observations at each iteration, which has only a minimal effect on the spectroscopic elements. These corrections range from -0.0145 to +0.0043 days (a change of 27 minutes over the $6.7 \mathrm{yr}$ time span of the more recent velocities) and are listed in Table 1.

Standard nonlinear least-squares techniques were used to solve simultaneously for the 19 unknowns that minimize the overall $\chi^{2}$ of the observations (Press et al. 1992, p. 650). Weights were assigned to the measurements according to their individual errors. Most of the older times of minimum have no published errors, so those were determined by iterations in order to achieve a reduced $\chi^{2}$ near unity for each type of observation (visual, photographic, photoelectric). The adopted errors are 0.021 days for photographic data, 0.011 days for the visual timings, and 0.0015 days for photoelectric/CCD minima. The published errors of the more recent $\mathrm{CCD}$ timings were found to require adjustments to achieve reduced $\chi^{2}$ values of unity. The scale factors used are 0.86 and 3.62 for the primary and secondary minima, respectively. The larger factor for the secondary is consistent with the smaller depth of that eclipse. The published uncertainties of the Hipparcos observations were found to require a minor adjustment by a factor of 0.85 , and the optimal errors for the radial velocity measurements were determined to be $1.51,5.40$, and $1.34 \mathrm{~km} \mathrm{~s}^{-1}$ for stars Aa, $\mathrm{Ab}$, and $\mathrm{B}$, respectively. A total of 332 observations were used: 75 radial velocities for each star, 43 times of primary eclipse, 6 times of secondary eclipse, 56 Hipparcos observations, and 2 parallax measurements (see above).

The results of this global fit are given in the third column of Table 10, including derived quantities such as the masses of the three stars. The uncertainties listed for all derived quantities take full account of correlations between different elements (offdiagonal elements of the covariance matrix). Initial solutions allowing for eccentricity in the inner orbit yielded a value not significantly different from zero, so a circular orbit was adopted. The outer orbit is moderately eccentric ( $e=0.469)$, and its period is determined to be $15.8 \mathrm{yr}$, with an error of only about $1.3 \%$.

The radial velocity observations and orbital solution for the inner eclipsing pair are shown graphically in Figure 9, with the motion in the outer orbit removed. In Figure $10 a$ we show the velocities of the third star as a function of time, along with the measured velocities of the center of mass of the inner binary and the computed curves for the outer orbit. The $O-C$ timing residuals (primary and secondary) from the best-fit linear ephemeris (Table 10) are displayed as a function of time in Figure 10b, for the more recent visual and photoelectric/CCD measurements. The solid line represents the computed third-body effect. The semiamplitude of this curve, as derived from our global fit, is $K_{O-C}=0.01458 \pm 0.00048$ days. Residuals from the curve for the individual timings are listed in Table 2. The time axis in both panels of Figure 10 is the same to show the complementarity of the data. In addition, the time location of the Hipparcos observations is shown in Figure $10 b$ and happens to be centered around periastron passage in the outer orbit.

The astrometric motion of the photocenter of V1061 Cyg on the plane of the sky is illustrated in Figure 11, where the axes are parallel to the right ascension and declination directions. Figure $11 a$ shows the total motion resulting from the combined effects of annual parallax, proper motion, and orbital motion. The dominant contribution is from the proper motion (nearly $40 \mathrm{mas} \mathrm{yr}^{-1}$ ), which is indicated with an arrow. Parallax and orbital motion are smaller and comparable effects. In Figure $11 b$ we have subtracted the proper-motion and parallactic contributions, leaving only the orbital motion with a $15.8 \mathrm{yr}$ period and a semimajor axis of 9.6 mas. The direction of motion (retrograde) is indicated by the arrow. The individual Hipparcos observations are depicted schematically in both panels of this figure but are seen more clearly in Figure $11 b$. Because they are one-dimensional in nature, the exact 

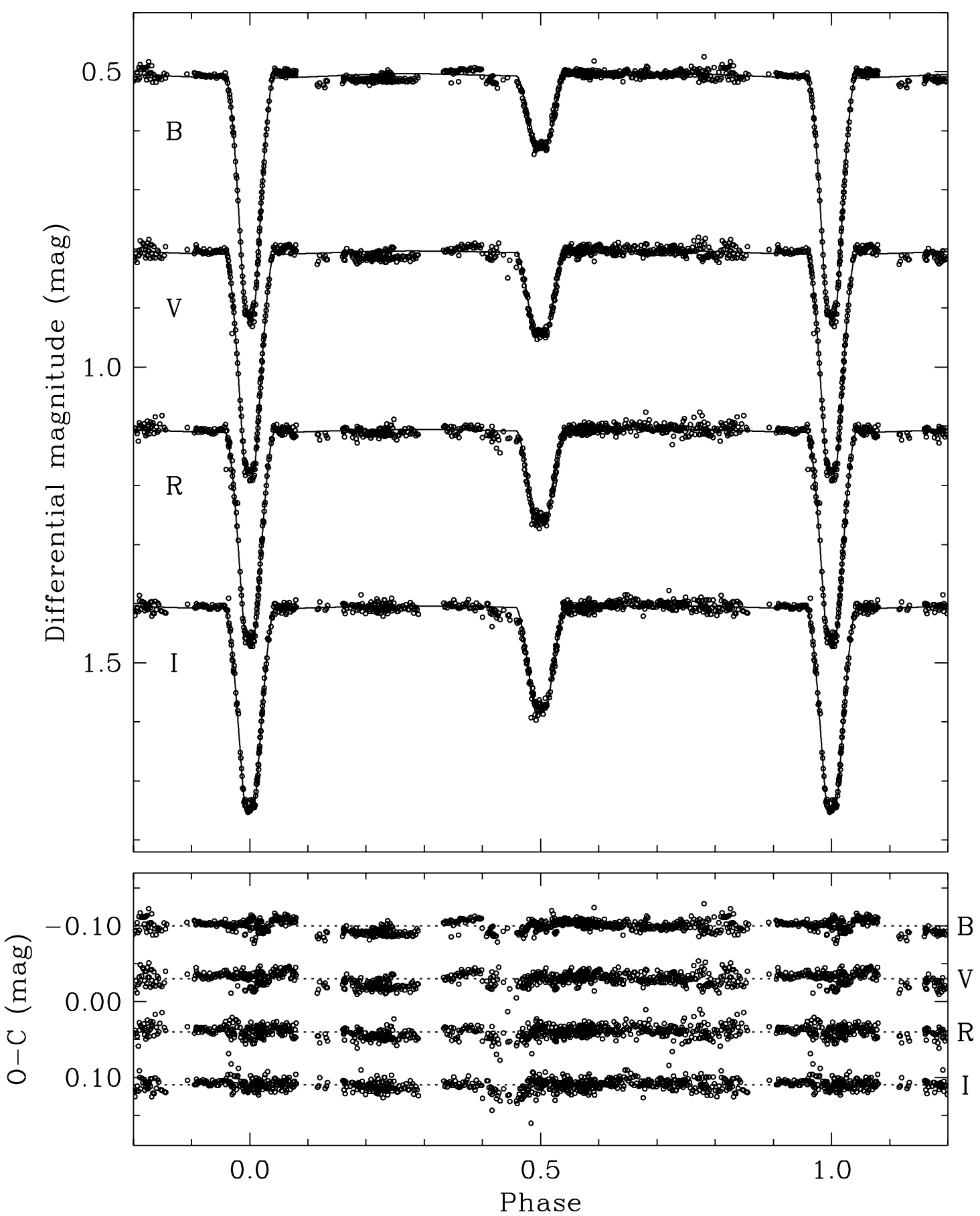

FIG. 7. - BVRI observations for V1061 Cyg from Gettysburg, along with our calculated light curves and residuals from those fits. The light curves in different passbands are shifted vertically for clarity. Patterns in the residuals near phase 0.45 may be due to spots (see text).

location of each measurement on the plane of the sky cannot be shown graphically. The filled circles represent the predicted location on the computed orbit. The dotted lines connecting to each filled circle indicate the scanning direction of the Hipparcos satellite for each measurement and show which side of the orbit the residual is on. The short line segments at the end of and perpendicular to the dotted lines indicate the direction along which the actual observation lies, although the precise location is undetermined. Occasionally more than one measurement was taken along the same scanning direction, in which case two or more short line segments appear on the same dotted lines. Figure $11 b$ shows that the observations are all on the long side of the orbit, and due to its orientation, they are roughly parallel to the direction of the proper motion. Since the orbit was not known at the time the Hipparcos catalog was released, we expect the orbital motion to have been absorbed into the published proper motion to some extent. Although the effect is not large, we do see a hint of this in that both components of the proper motion as published are larger than the values we derive from our global solution, by about 2.5 and $4.0 \mathrm{mas} \mathrm{yr}^{-1}$ for $\mu_{\alpha}^{*}$ and $\mu_{\delta}$, respectively. Statistically the effect is somewhat more significant in declination.

\section{ABSOLUTE DIMENSIONS}

The combination of the photometric results in $\S 2.3$ and the spectroscopic results in $\S 3$ yields the absolute masses and radii of the components of the eclipsing binary, which we list in 

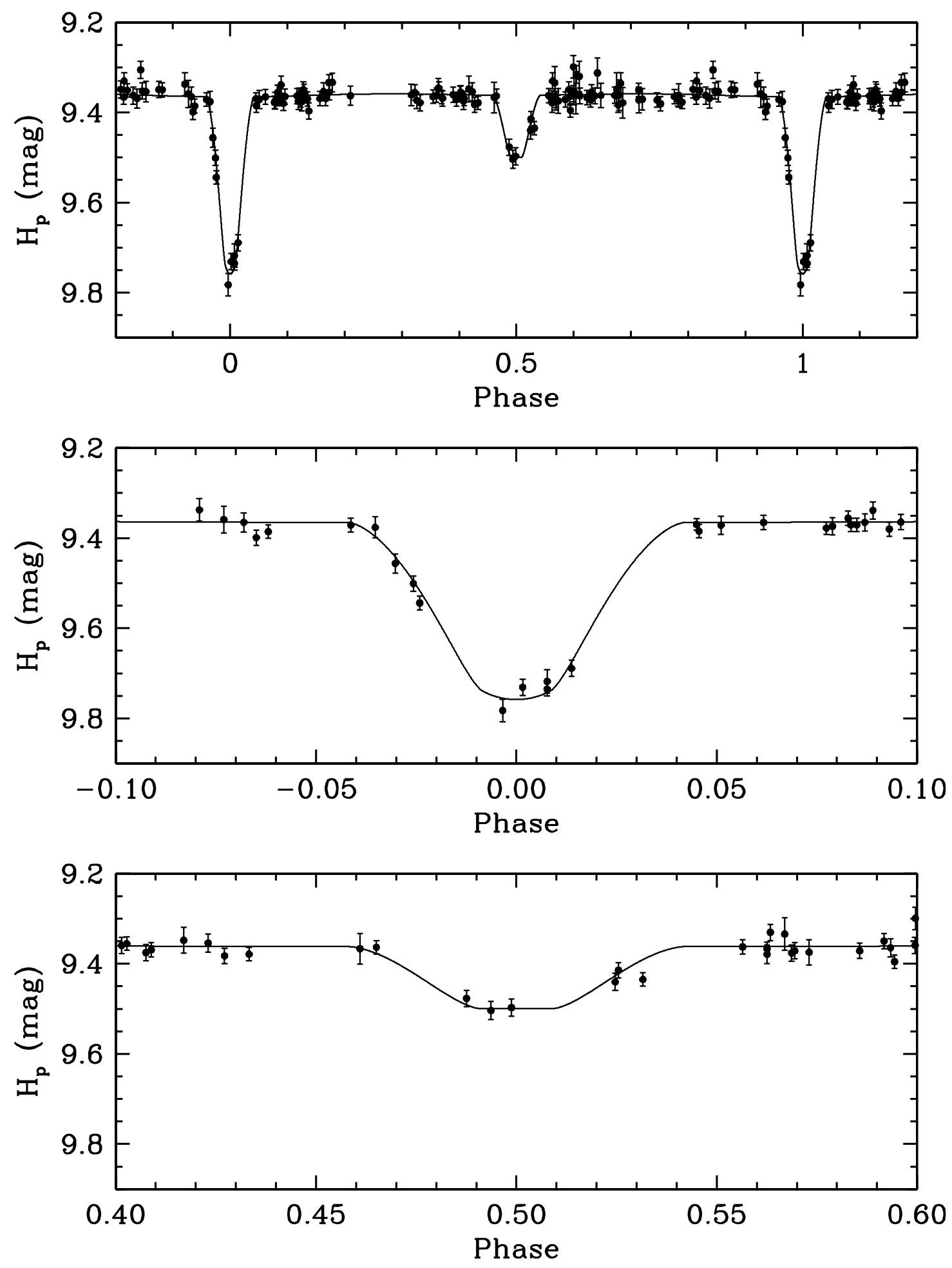

FIG. 8. - Hipparcos photometric observations for V1061 Cyg along with our light-curve fit using EBOP. The bottom two panels show enlargements of the regions around the minima. This fit was used to derive the fractional luminosity of star B in the $H_{p}$ passband (see text).

Table 11 along with other properties, as well as inferred parameters for the third star (see $\S 5.3$ ). For the photometric results we have adopted the weighted average of the Gettysburg and URSA elements, which are $r_{\mathrm{Aa}}=0.1668 \pm 0.0017, k=0.598 \pm 0.004$, and $i_{\mathrm{A}}=88^{\circ} .2 \pm 0.4$. The precision of the absolute masses of the binary components is $1.2 \%$ for star $\mathrm{Aa}$ and $0.7 \%$ for $\mathrm{Ab}$, while that of star B is only $4 \%$. The absolute radii are determined to $1 \%$ and $2 \%$ for the primary and secondary, respectively. The measured projected rotational velocities of both stars are in excellent agree- ment with the values computed for synchronous rotation, as expected for such a short period.

The parallax determination is much improved compared to the original Hipparcos result, the error being reduced from $17 \%$ to about 3\% driven mostly by the individual estimates for stars Aa and $\mathrm{Ab}$ based on their physical properties, and to some extent also by the astrometry. The increased accuracy has an impact on the comparison with theory described below. The corresponding distance to the system is $166.9 \pm 5.6 \mathrm{pc}$. The space motion of 
TABLE 10

Orbital Solutions for V1061 Cyg

\begin{tabular}{|c|c|c|}
\hline Parameter & Spectroscopic Only & Combined \\
\hline \multicolumn{3}{|l|}{ Adjusted quantities from inner orbit (Aa and $\mathrm{Ab}$ ): } \\
\hline$P_{\mathrm{A}}$ (days) & $2.3466487 \pm 0.0000049$ & $2.34665473 \pm 0.00000035$ \\
\hline 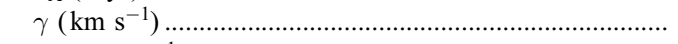 & $-7.84 \pm 0.25$ & $-5.67 \pm 0.14$ \\
\hline$K_{\mathrm{Aa}}\left(\mathrm{km} \mathrm{s}^{-1}\right)$ & $87.96 \pm 0.34$ & $87.83 \pm 0.20$ \\
\hline 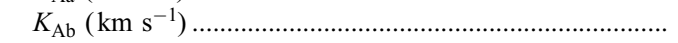 & $120.54 \pm 0.73$ & $120.87 \pm 0.70$ \\
\hline$e_{\mathrm{A}}$ & 0 (fixed) & 0 (fixed) \\
\hline$\omega_{\mathrm{Aa}}(\operatorname{deg}) \ldots \ldots \ldots \ldots \ldots \ldots \ldots \ldots \ldots \ldots \ldots$ & $\ldots$ & $\ldots$ \\
\hline 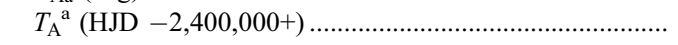 & $52015.9050 \pm 0.0018$ & $52015.89295 \pm 0.00058$ \\
\hline \multicolumn{3}{|l|}{ Adjusted quantities from outer orbit $(\mathrm{Aa}+\mathrm{Ab}$ and $\mathrm{B})$ : } \\
\hline 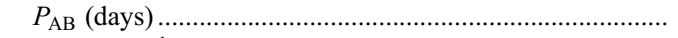 & $\ldots$ & $5786 \pm 76$ \\
\hline 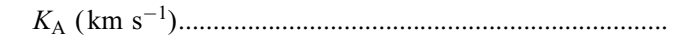 & $\ldots$ & $5.37 \pm 0.26$ \\
\hline$e_{\mathrm{AB}}$ & $\ldots$ & $0.469 \pm 0.032$ \\
\hline 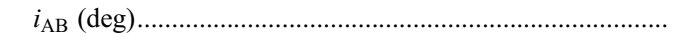 & $\ldots$ & $112.0 \pm 4.9$ \\
\hline$\omega_{\mathrm{A}}(\mathrm{deg})$ & $\ldots$ & $97.2 \pm 2.8$ \\
\hline$\Omega_{\mathrm{AB}}(\operatorname{deg}) \ldots \ldots \ldots \ldots \ldots \ldots \ldots \ldots$ & $\ldots$ & $27 \pm 44$ \\
\hline$T_{\mathrm{AB}}^{\mathrm{b}}(\mathrm{HJD}-2,400,000+) \ldots \ldots \ldots \ldots \ldots$ & $\cdots$ & $48,545 \pm 55$ \\
\hline \multicolumn{3}{|l|}{ Other adjusted quantities: } \\
\hline 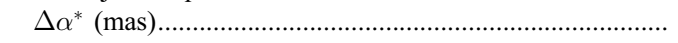 & $\cdots$ & $+1.2 \pm 1.9$ \\
\hline$\Delta \delta$ (mas) & $\ldots$ & $+0.40 \pm 0.67$ \\
\hline$\Delta \mu_{\alpha}^{*}\left(\operatorname{mas} \mathrm{yr}^{-1}\right)$ & $\ldots$ & $-2.5 \pm 3.5$ \\
\hline 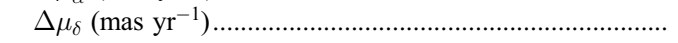 & $\cdots$ & $-4.0 \pm 1.9$ \\
\hline$\Delta \pi(\operatorname{mas})$ & $\ldots$ & $-0.26 \pm 0.20$ \\
\hline \multicolumn{3}{|l|}{ Derived quantities: } \\
\hline$K_{\mathrm{B}}\left(\mathrm{km} \mathrm{s}^{-1}\right) \ldots \ldots \ldots \ldots$ & $\ldots$ & $12.85 \pm 0.53$ \\
\hline$K_{O-C}($ days $) \ldots \ldots$ & $\ldots$ & $0.01458 \pm 0.00048$ \\
\hline$\mu_{\alpha}^{*}\left(\operatorname{mas} \mathrm{yr}^{-1}\right) \ldots \ldots$ & $\cdots$ & $+17.4 \pm 3.5$ \\
\hline$\mu_{\delta}\left(\operatorname{mas} \mathrm{yr}^{-1}\right)$ & $\ldots$ & $+35.5 \pm 1.9$ \\
\hline$\pi$ (mas) & $\ldots$ & $5.99 \pm 0.20$ \\
\hline 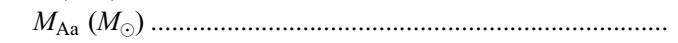 & $(1.274 \pm 0.018) / \sin ^{3} i_{\mathrm{A}}$ & $1.282 \pm 0.015$ \\
\hline$M_{\mathrm{Ab}}\left(M_{\odot}\right)$ & $(0.9297 \pm 0.0096) / \sin ^{3} i_{\mathrm{A}}$ & $0.9315 \pm 0.0068$ \\
\hline$M_{\mathrm{B}}\left(M_{\odot}\right)$ & $\cdots$ & $0.925 \pm 0.036$ \\
\hline 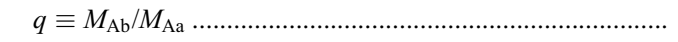 & $0.7297 \pm 0.0053$ & $0.7266 \pm 0.0042$ \\
\hline 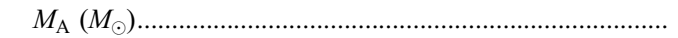 & $\ldots$ & $2.213 \pm 0.021$ \\
\hline 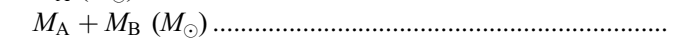 & $\ldots$ & $3.139 \pm 0.046$ \\
\hline$a_{\mathrm{A}}\left(R_{\odot}\right)$ & $(9.666 \pm 0.038) / \sin i_{\mathrm{A}}$ & $9.681 \pm 0.031$ \\
\hline$a_{\mathrm{AB}}^{\prime \prime}(\mathrm{mas})$ & $\ldots$ & $55.3 \pm 1.8$ \\
\hline$a_{\mathrm{AB}}(\mathrm{AU})$ & $\ldots$ & $9.235 \pm 0.082$ \\
\hline$\alpha_{\mathrm{ph}}^{\prime \prime}(\mathrm{mas}) \ldots \ldots$ & $\cdots$ & $9.55 \pm 0.54$ \\
\hline
\end{tabular}

a Time of primary eclipse.

b Time of periastron passage.

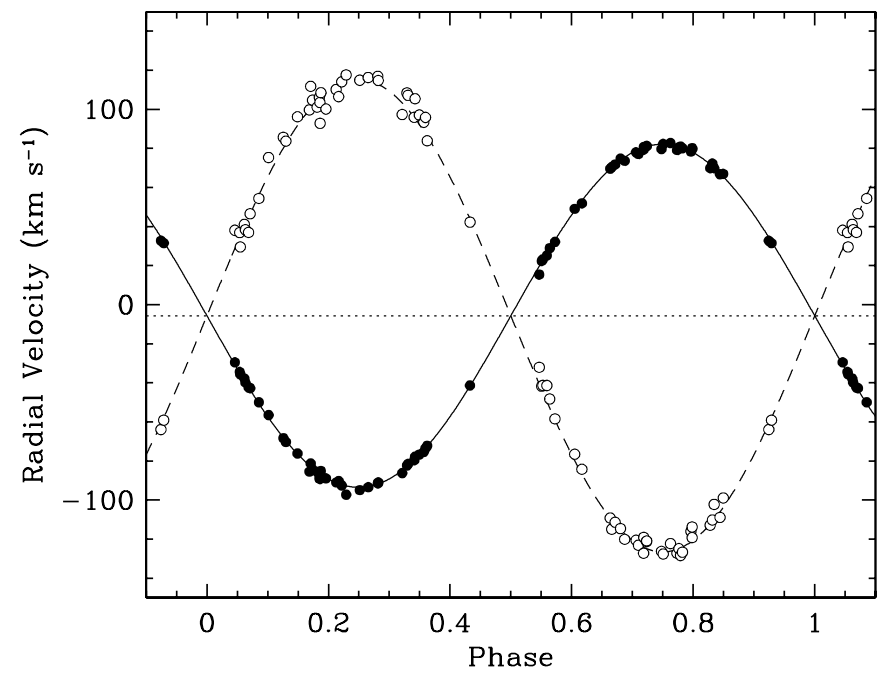

FIG. 9.- Radial velocity observations for stars Aa ( filled circles) and Ab (open circles) in the eclipsing binary, with our fitted orbit. The motion of the binary in the wide orbit has been subtracted. Phase 0.0 corresponds to the primary eclipse, and the dotted line represents the center-of-mass velocity of the system.
V1061 Cyg in the Galactic frame is $U=-20 \mathrm{~km} \mathrm{~s}^{-1}, V=$ $-2 \mathrm{~km} \mathrm{~s}^{-1}$, and $W=+16 \mathrm{~km} \mathrm{~s}^{-1}$ (relative to the local standard of rest), quite typical of Population I stars. The inclination angles of the inner and outer orbits $\left(i_{\mathrm{A}}\right.$ and $\left.i_{\mathrm{AB}}\right)$ are known from our spectroscopic and light-curve analyses, but the relative inclination $\phi$ between the two orbits cannot be determined because the position angle of the node for the eclipsing pair is unknown. ${ }^{8}$ However, a lower limit of $\phi_{\min }=20^{\circ} .2 \pm 4^{\circ} .9$ can be placed, which appears to exclude coplanarity. The semimajor axis of the photocenter of the binary in the wide orbit is only 9.55 mas, but the relative semimajor axis is much larger (55.3 mas). Considering that the orbit is eccentric and therefore that the angular separation can be as large as 80 mas, the third star is potentially resolvable with current techniques (e.g., speckle interferometry on $4 \mathrm{~m}$ class telescopes, or adaptive optics on 8-10 m class telescopes) despite being 2 mag fainter than the binary in the

${ }^{8}$ The relative inclination angle of the two orbits is given by $\cos \phi=$ $\cos i_{\mathrm{A}} \cos i_{\mathrm{AB}}+\sin i_{\mathrm{A}} \sin i_{\mathrm{AB}} \cos \left(\Omega_{\mathrm{A}}-\Omega_{\mathrm{AB}}\right)$ (e.g., Fekel 1981). Since $\Omega_{\mathrm{A}}$ is unknown, we can only set limits to $\cos \left(\Omega_{\mathrm{A}}-\Omega_{\mathrm{AB}}\right)$ between -1 and +1 , which leads to $i_{\mathrm{A}}-i_{\mathrm{AB}} \leq \phi \leq i_{\mathrm{A}}+i_{\mathrm{AB}}$. 


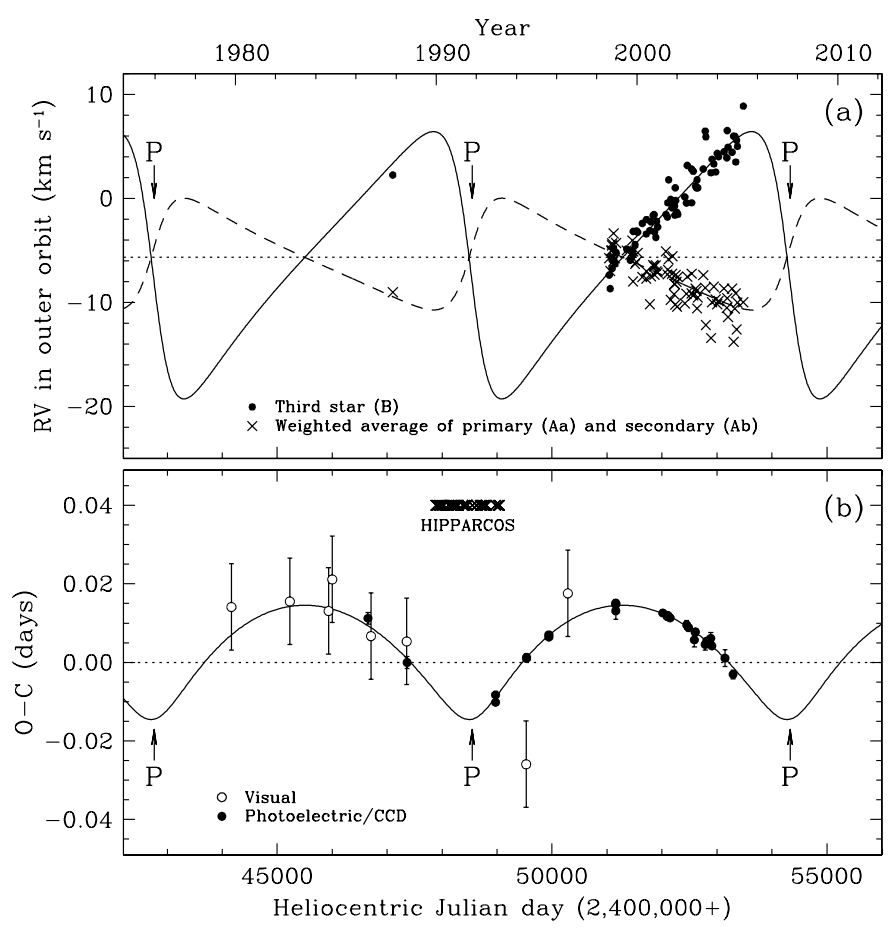

FIg. 10.- Radial velocities for V1061 Cyg in the outer orbit, and $O-C$ timing residuals as a function of time. The curves represent our best-fit model that uses velocities simultaneously with the eclipse timings and Hipparcos observations. (a) Velocities for the third star ( filled circles) and the center of mass of the inner binary (crosses). The latter is computed at each date from the weighted average of the two stars, after removing the motion in the inner orbit. The center-of-mass velocity is indicated with the dotted line. (b) $O-C$ diagram of the more recent eclipse timings based on the linear ephemeris in Table 10. Open circles are for visual measurements, and filled circles for photoelectric/ CCD measurements. The older photographic timings have much larger error bars and are not shown, for clarity. The dates of the Hipparcos observations are indicated and happen to cover periastron passage (arrows).

visible. Maximum separation should occur around the year 2014.

The central surface brightness parameter from the light-curve solutions constrains the difference between the effective temperatures of the stars in the binary. To estimate the mean temperature of the system, we made use of absolute $U B V$ photometry outside of eclipse available from the work of Lacy (1992), the $B_{T}$ and $V_{T}$ magnitudes from the Tycho-2 catalog (Høg et al. 2000), and the $J H K_{s}$ magnitudes from the Two Micron All Sky Survey (2MASS) catalog. Color indices from various combinations of these magnitudes along with calibrations by Alonso et al. (1996) and Ramírez \& Meléndez (2005) lead to a mean temperature close to $6000 \mathrm{~K}$, similar to our estimate in $\S 2.3$. Folding in the spectroscopic estimates, we obtain average values for the primary and secondary of $6180 \pm 100$ and $5300 \pm 150 \mathrm{~K}$, respectively, which we adopt here. These correspond to spectral types of approximately F9 and G8, while the third star is roughly G4 (Cox 2000, p. 388).

\section{DISCUSSION}

The high precision of the mass and radius determinations for stars $\mathrm{Aa}$ and $\mathrm{Ab}$ (errors of $1 \%-2 \%$ ), along with the subsolar secondary mass and a mass ratio that is quite different from unity $(q=0.7266)$, makes V1061 Cyg a particularly valuable system to test models of single-star evolution. The four measured properties available for testing are the mass, radius, effective temperature, and luminosity $\left(M_{V}\right)$. The latter two are of course related through the Stefan-Boltzmann law, although they were determined independently here since we know the distance. Because
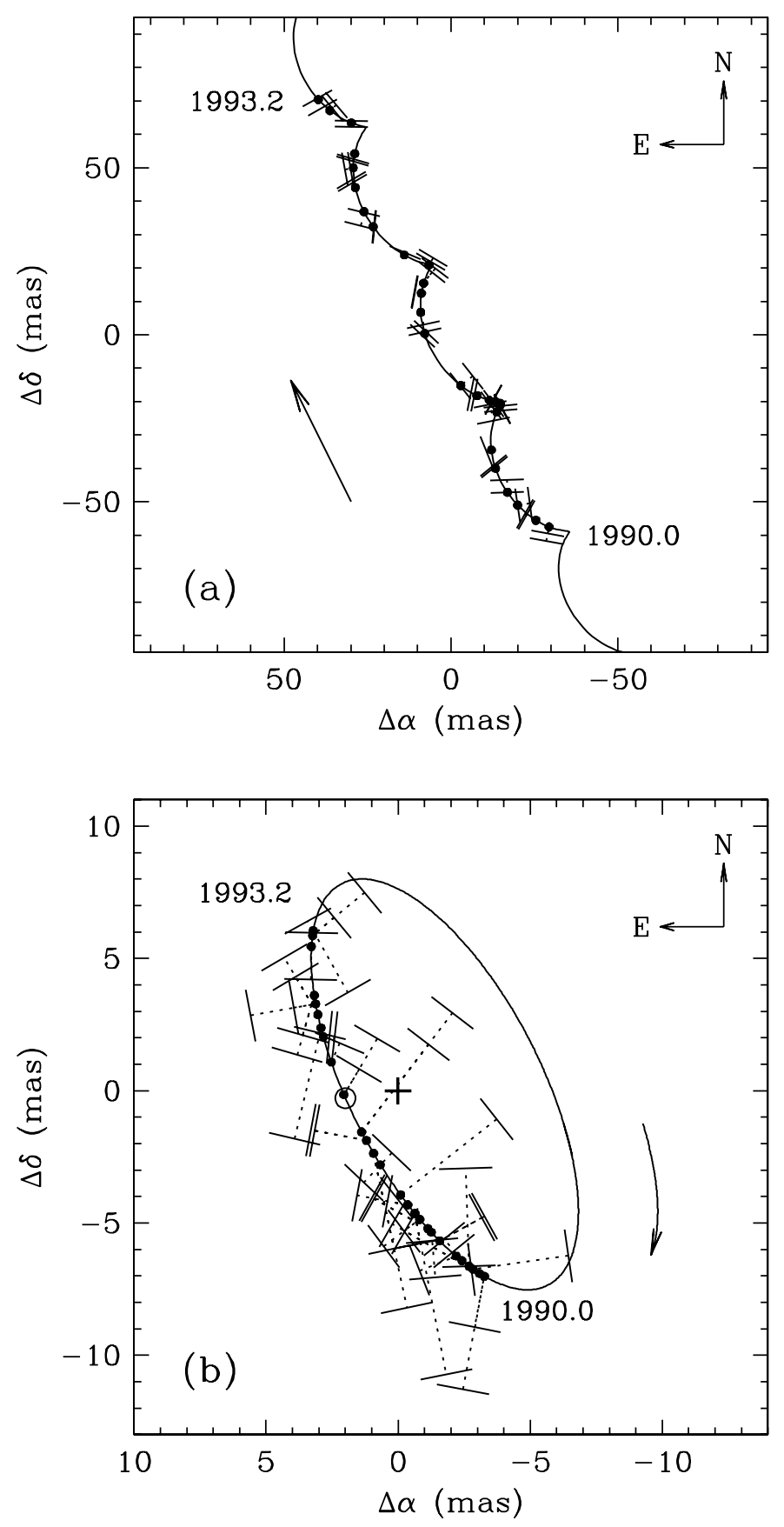

FIG. 11.-Path of the center of light of V1061 Cyg on the plane of the sky, along with the Hipparcos observations (abscissa residuals). See text for an explanation of the graphical representation of these one-dimensional measurements. (a) Total motion resulting from the combined effects of parallax, proper motion, and orbital motion according to the global solution described in the text. The arrow indicates the direction and magnitude of the annual proper motion. (b) Residual orbital motion after subtracting the parallactic and proper-motion contributions. The plus sign represents the center of mass of the triple, and the direction of motion in the orbit (retrograde) is indicated by the arrow. Periastron is shown with an open circle.

the temperatures were derived somewhat more indirectly and have greater uncertainties than $M_{V}$, we have preferred to rely here on the absolute visual magnitudes, inferred from the apparent brightness of the system (ignoring extinction; see $\S 2.3$ ), the fractional luminosities, and the parallax.

No accurate estimate of the metallicity is available. The Population I kinematics $(\S 4)$ provides only circumstantial evidence that the composition is perhaps near solar. In the following we have made that assumption initially, although nonsolar compositions 
TABLE 11

Physical Parameters for V1061 Cyg

\begin{tabular}{|c|c|c|c|}
\hline Parameter & Primary & Secondary & Tertiary $^{\mathrm{a}}$ \\
\hline 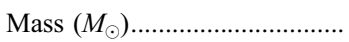 & $1.282 \pm 0.015$ & $0.9315 \pm 0.0068$ & $0.925 \pm 0.036$ \\
\hline 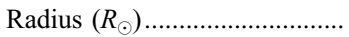 & $1.615 \pm 0.017$ & $0.974 \pm 0.020$ & $0.870 \pm 0.087$ \\
\hline $\log g$ & $4.129 \pm 0.011$ & $4.430 \pm 0.018$ & $4.525 \pm 0.088$ \\
\hline 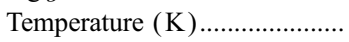 & $6180 \pm 100$ & $5300 \pm 150$ & $5670 \pm 150$ \\
\hline 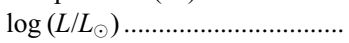 & $0.533 \pm 0.030$ & $-0.173 \pm 0.052$ & $-0.153 \pm 0.066$ \\
\hline 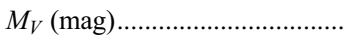 & $3.456 \pm 0.074$ & $5.457 \pm 0.079$ & $5.23 \pm 0.16$ \\
\hline$v \sin i\left(\mathrm{~km} \mathrm{~s}^{-1}\right) \ldots \ldots \ldots \ldots \ldots \ldots \ldots \ldots \ldots \ldots \ldots$ & $36 \pm 2$ & $20 \pm 3$ & $2 \pm 3$ \\
\hline$v_{\mathrm{sync}} \sin i\left(\mathrm{~km} \mathrm{~s}^{-1}\right) \ldots \ldots \ldots \ldots \ldots \ldots$ & $34.8 \pm 0.4$ & $21.0 \pm 0.4$ & $\ldots$ \\
\hline 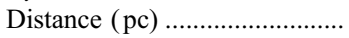 & $166.9 \pm 5.6$ & $166.9 \pm 5.6$ & $166.9 \pm 5.6$ \\
\hline 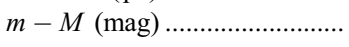 & $6.113 \pm 0.073$ & $6.113 \pm 0.073$ & $6.113 \pm 0.073$ \\
\hline
\end{tabular}

a The radius, $\log g$, and luminosity of the tertiary were inferred from $M_{V}$, temperature, and bolometric corrections by Lejeune et al. (1998).

have also been explored by leaving the metallicity as a free parameter. In principle, this additional freedom may appear to weaken the test; however, in this particular case it has no effect on our main conclusion, as seen below.

\subsection{Comparison with Evolutionary Models}

In Figure 12 we display the radius and absolute visual magnitude of V1061 Cyg as a function of mass, along with theoretical isochrones from the Yonsei-Yale series of evolutionary models (Yi et al. 2001; Demarque et al. 2004) for solar metallicity $\left(Z_{\odot} \equiv\right.$ 0.01812). As do most models, these treat convection in the mixing-length approximation and adopt a mixing-length parameter that best fits the properties of the Sun $\left(\alpha_{\mathrm{ML}}=1.7432\right)$. The color transformations and bolometric corrections adopted are from the tables by Lejeune et al. (1998). The figure shows that, within the errors, a 3.4 Gyr isochrone for this metallicity provides an excellent match to all observations except for the radius of the secondary, which is $\sim 10 \%$ larger than predicted. This very significant difference is 5 times the uncertainty. Since the luminosity of the secondary is well reproduced by the models, it follows that the effective temperature must be cooler than predicted. Indeed, our estimate from $\S 4$ is $T_{\text {eff }}^{\mathrm{Ab}}=5300 \pm 150 \mathrm{~K}$, formally cooler than the predicted value of $5460 \mathrm{~K}$ but only at the $1 \sigma$ level. A more precise estimate of the secondary temperature may be derived from our $M_{V}$ and the radius of the star (with bolometric corrections taken also from Lejeune et al. [1998], for consistency). This gives $T_{\mathrm{eff}}^{\mathrm{Ab}}=5210 \pm 80 \mathrm{~K}$, which is lower than the models predict at a more significant $3.1 \sigma$ level. Thus, the secondary does appear to be too cool by roughly $200 \mathrm{~K}$.

Extensive tests show that adjustments in the age or metallicity of the isochrones do not improve the agreement. Figure $13 a$ illustrates this in the age/metallicity plane. The shaded areas represent all isochrones that are consistent with the measured mass, radius, and $M_{V}$ of each star within the estimated uncertainties. The shaded areas do not overlap, meaning that no single isochrone can reproduce all properties of both stars simultaneously. The region spanned by models that are consistent with the primary properties $([\mathrm{Fe} / \mathrm{H}]$ within \pm 0.14 dex of solar, ages of $3.06-$ $3.78 \mathrm{Gyr})$ is shown in the mass/radius and mass $/ M_{V}$ diagrams in Figures $13 b$ and $13 c$. None of those models come close to matching the radius of the secondary star, although they do agree well with its absolute magnitude, as stated above.

As a test we repeated the comparison using a different set of isochrones by Girardi et al. (2000), from the Padova group. In these models the adopted mixing-length parameter that best reproduces the $\operatorname{Sun}\left(\right.$ for $Z_{\odot} \equiv 0.019$ ) is $\alpha_{\mathrm{ML}}=1.68$, and a number of other physical ingredients are somewhat different, as are the color transformations and bolometric corrections. Nevertheless, the fits to V1061 Cyg are very similar and again indicate a large discrepancy in the secondary radius $R_{\mathrm{Ab}}$ and a smaller difference in temperature.

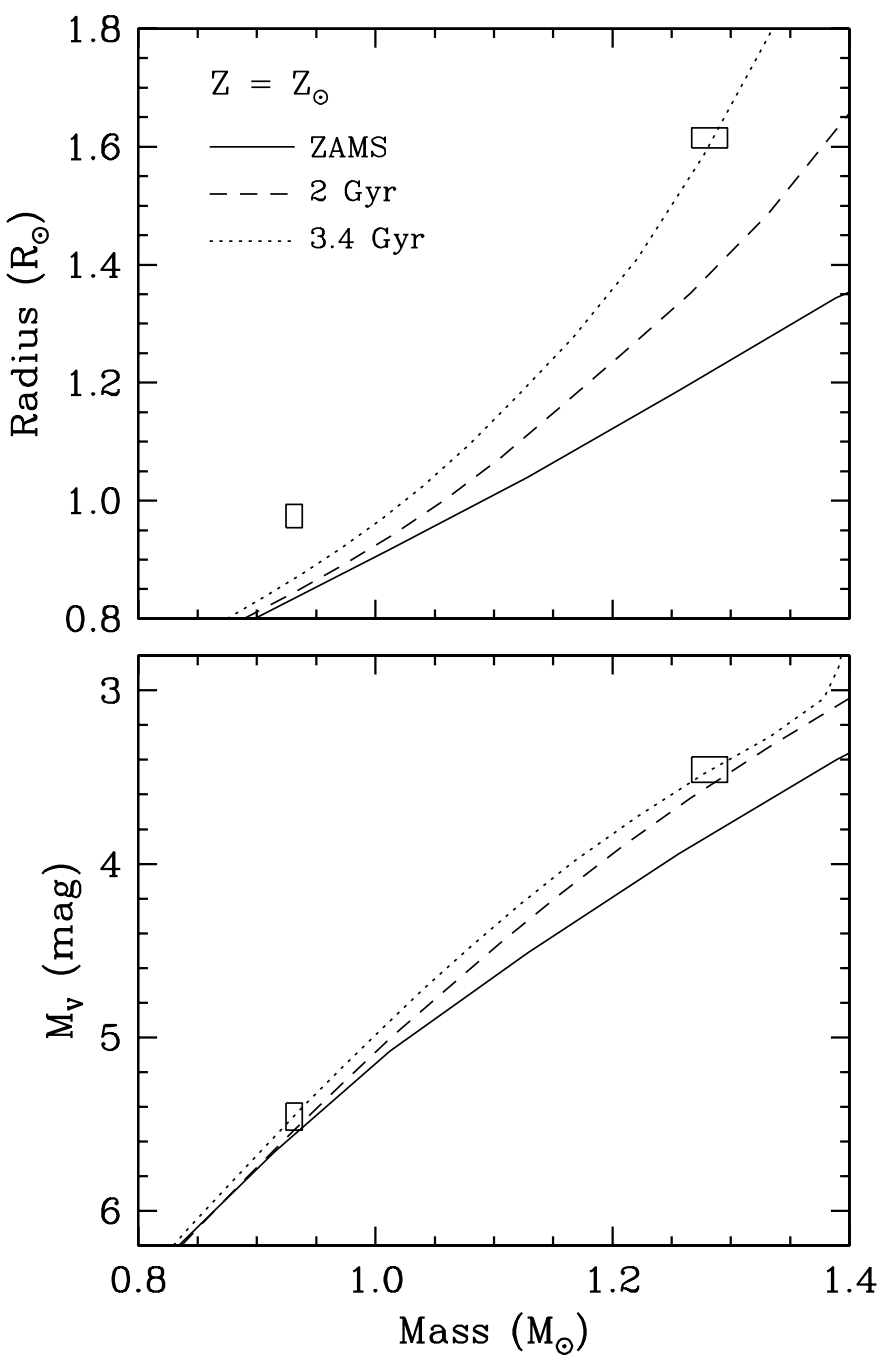

FIG. 12.-Comparison between the observed properties (error boxes) of the primary and secondary of V1061 Cyg (mass, radius, absolute visual magnitude) and model isochrones by Yi et al. (2001) and Demarque et al. (2004) for solar metallicity and three different ages, as labeled. 

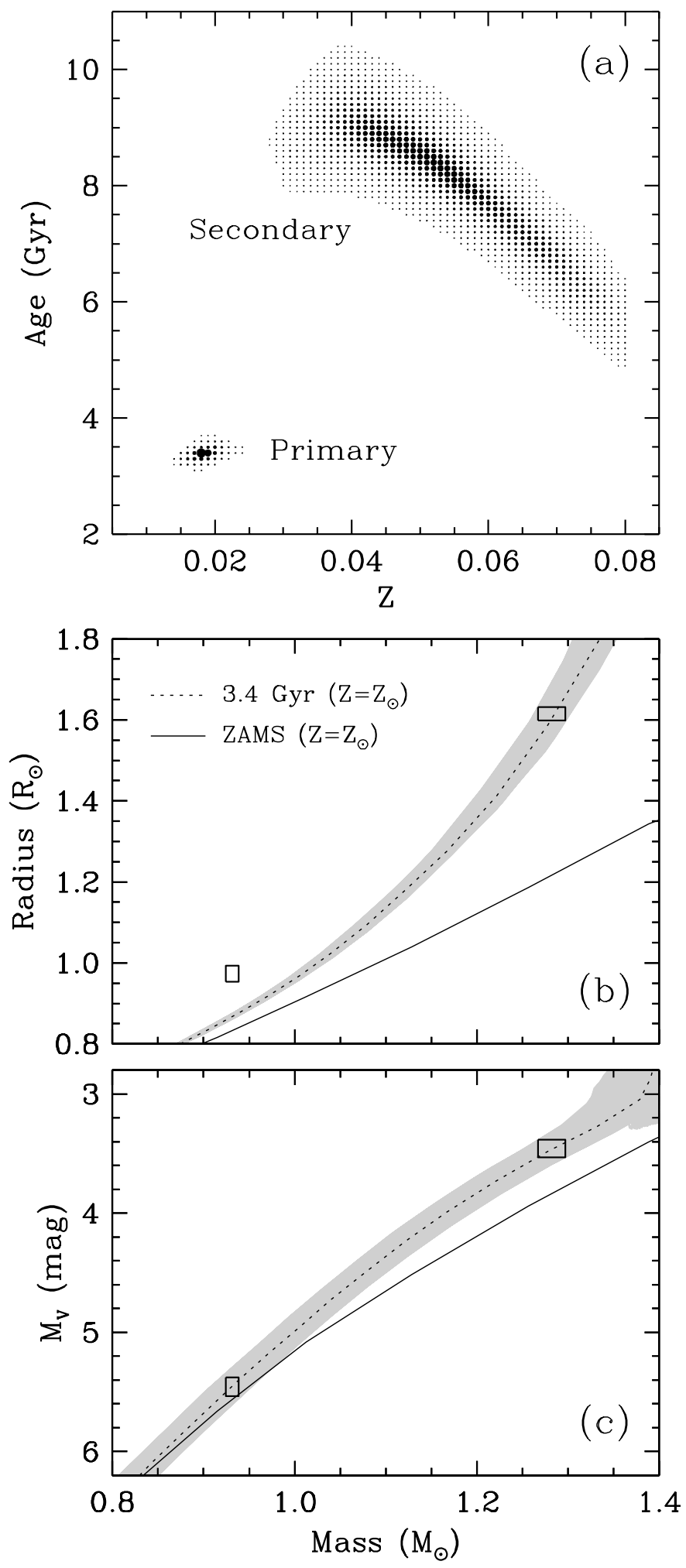

FIG. 13.-Age and metallicity range of theoretical models by Yi et al. (2001) and Demarque et al. (2004) that fit the measured properties of the primary and secondary components of V1061 Cyg. (a) Shaded areas represent age-metallicity combinations consistent with the measured mass, radius, and absolute visual magnitude of each star. The gray-scale level is a measure of how well each model matches the exact values of $M, R$, and $M_{V}$ (darker areas represent a better fit). (b) The shaded area represents the projection onto the mass/radius diagram of all models indicated in $(a)$ that fit the primary star within its error boxes. None of the models come close to reproducing the secondary radius. (c) Same as above, for the mass $/ M_{V}$ plane. The secondary star is well fitted by the same models that match the primary.
Both of the above sets of evolutionary models adopt a gray approximation for the outer boundary conditions to the internal structure equations. More sophisticated nongray atmospheres have been used in the models by Siess et al. (1997) and Baraffe et al. (1998), which has been shown to be important for low-mass stars (see, e.g., Chabrier \& Baraffe 1997). In addition, these models incorporate an improved equation of state and other refinements, although all this should only have an impact for stars considerably smaller than the secondary of V1061 Cyg (which is near the calibration point at $\left.1 M_{\odot}\right)$. Indeed, the comparison between V1061 Cyg and the Baraffe et al. (1998) isochrones $\left(Z_{\odot} \equiv\right.$ $0.020, \alpha_{\mathrm{ML}}=1.9$ ) is not very different from the two previous fits and yields a similar age as before based on the agreement with all observed properties except $R_{\mathrm{Ab}}$.

The measured radii depend mainly on the results from the lightcurve fits and to a lesser degree on the spectroscopy. As described earlier, tests carried out during the analysis of both types of data make it very unlikely that either set of results is biased enough to explain the discrepancy. Since the properties of the more massive primary star appear to be well reproduced by the models (as expected from previous experience; see Andersen 2003), suspicion falls on our theoretical understanding of stars under a solar mass. Similar evidence has been presented over the last several years (e.g., Popper 1997; Clausen et al. 1999a; Torres \& Ribas 2002; Ribas 2003; Dawson \& De Robertis 2004; López-Morales \& Ribas 2005), but in fact indications go as far back as the work by Hoxie (1973), Lacy (1977), and others. All of these studies have shown that theoretical calculations for stars less massive than the Sun tend to underestimate the radius by as much as $10 \%-20 \%$. Temperature differences also appear to be present, as in V1061 Cyg.

The models by Baraffe et al. (1998) we used above are also available for lower values of the mixing-length parameter in the mass range of interest. In Figure 14 we compare the observations for V1061 Cyg against solar-metallicity models for $\alpha_{\mathrm{ML}}=1.0$ and 1.5 , in addition to $\alpha_{\mathrm{ML}}=1.9$, adjusting the age to satisfy the constraints on the primary star within the errors. A 2.6 Gyr isochrone with $\alpha_{\mathrm{ML}}=1.0$ provides a significantly better fit to the secondary radius than the one with $\alpha_{\mathrm{ML}}=1.9$ used earlier, although the model prediction still falls short of the observed $R_{\mathrm{Ab}}$ value by about $1.8 \sigma$ at the measured mass. The reduced convection implied by the lower mixing-length parameter in these models compared to the previous ones has the effect of increasing the theoretical radii and slightly lowering the effective temperatures. The predicted temperature for $\alpha_{\mathrm{ML}}=1.0$ and $M=M_{\mathrm{Ab}}$ is $5110 \mathrm{~K}$, somewhat closer than before to the empirical result of $5210 \mathrm{~K}$ (see above).

The considerably smaller value of $\alpha_{\mathrm{ML}}$ apparently required by a star so close to the solar mass as V1061 Cyg Ab is perhaps somewhat surprising, although other studies have found similar evidence in some stars and have even argued for a mass dependence, with smaller values of $\alpha_{\mathrm{ML}}$ for later type stars (e.g., Lastennet et al. 2003). However, the observational evidence for this is often contradictory (see Eggenberger et al. 2004). Theoretical studies, on the other hand, have tended to predict the opposite dependence with mass (Ludwig et al. 1999; Trampedach et al. 1999).

\subsection{Comparison with Other Stars: The Activity-Radius Connection}

Further clues on these disagreements may be gained from looking at other eclipsing binary systems with well-determined properties. To avoid any possible dependence on mass, we focus here on binaries having at least one star in the same mass range as the secondary of V1061 Cyg. Three such systems are available with reliable determinations of the mass, radius, and temperature 

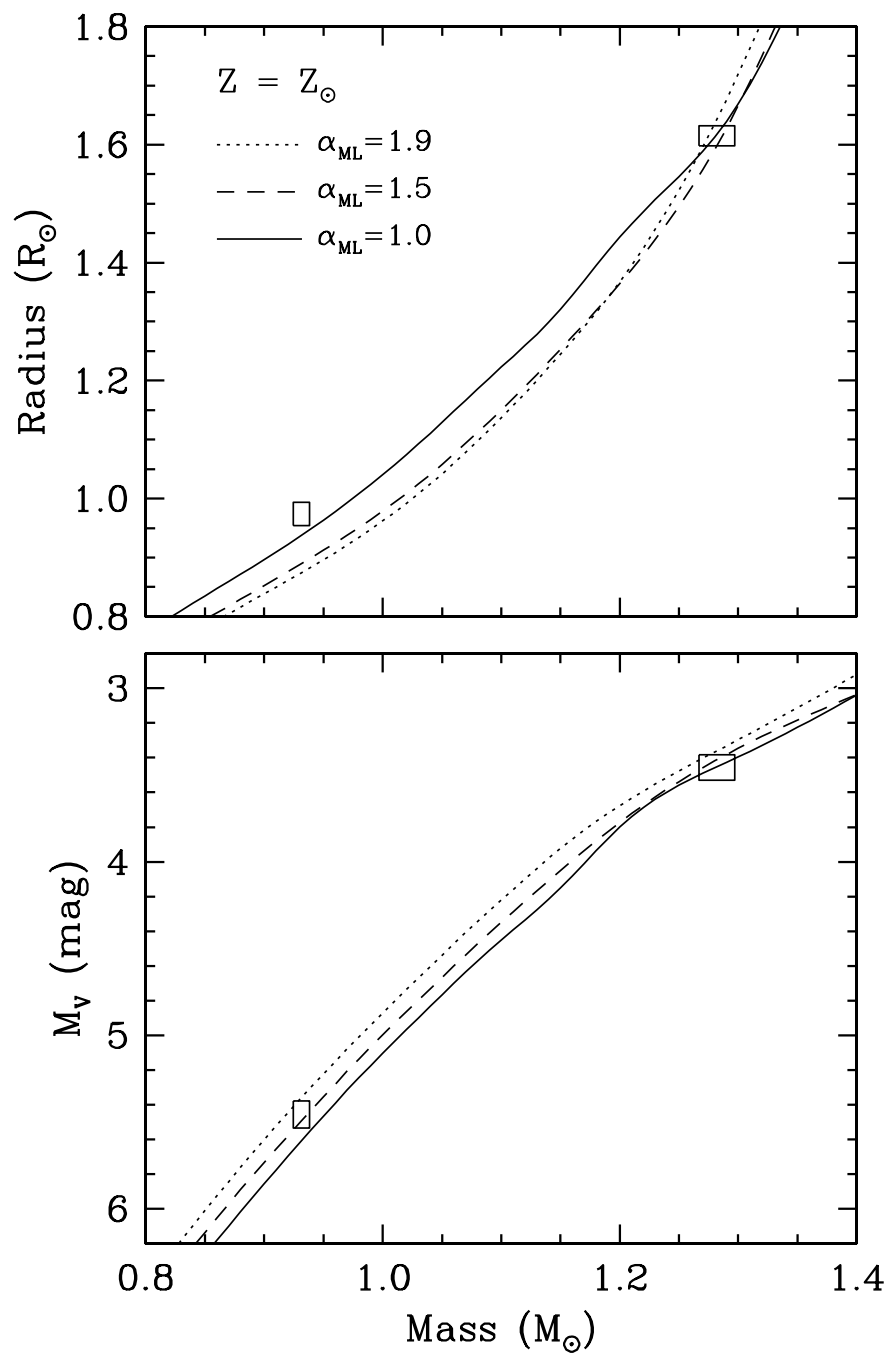

FIG. 14.-Observations of V1061 Cyg (error boxes) compared against model isochrones by Baraffe et al. (1998) for three different values of the mixing-length parameter, as labeled. Solar metallicity is assumed, and the age of each isochrone has been tuned to fit the properties of the primary as closely as possible within errors (2.6 Gyr for $\alpha_{\mathrm{ML}}=1.0,3.1 \mathrm{Gyr}$ for $\alpha_{\mathrm{ML}}=1.5$, and $3.4 \mathrm{Gyr}$ for $\alpha_{\mathrm{ML}}=1.9$ ). The model with $\alpha_{\mathrm{ML}}=1.0$ (solid line) is seen to provide a much closer match to the radius of the secondary than the one with $\alpha_{\mathrm{ML}}$ adjusted to fit the Sun.

good to better than 3\%: RW Lac (Lacy et al. 2005), in which the primary is within $0.5 \%$ of the mass of V1061 Cyg Ab; FL Lyr, with a secondary only $3 \%$ more massive; and HS Aur, with a primary that is $3.5 \%$ less massive. The latter two are from work by Popper et al. (1986) and Andersen (1991), and FL Lyr has the added advantage that the components have quite dissimilar masses (as in the case of V1061 Cyg), which provides increased leverage for the test. The main properties of these systems are collected in Table 12, along with V1061 Cyg and the Sun for reference. Two additional systems (CG Cyg and RT And; Popper 1994) have one component in the same mass range, but the temperature determinations are considerably more uncertain and are thus less useful for our purposes.

We compared the measurements for RW Lac, FL Lyr, and HS Aur to the Yonsei-Yale models in the same way we did for V1061 Cyg, adjusting the age and metallicity of the isochrones to obtain the best possible fit (see Fig. 15). FL Lyr is seen to present the same problem as V1061 Cyg: the secondary appears too large compared to theory, by about the same amount as we saw before $(\sim 10 \%)$, while the properties of the primary are well fitted. On the other hand, the primary of RW Lac is virtually identical in mass to V1061 Cyg Ab, yet it shows no indication of a radius discrepancy. The primary in HS Aur also seems to be well reproduced by the models (as well as the secondary), within the errors.

A pattern that may explain why stars of very similar mass sometimes appear too large, while other times they conform well to theory, is seen in the activity level they present. Both V1061 Cyg $\mathrm{Ab}$ and FL Lyr B, which show the radius discrepancy, are in relatively tight systems with orbital periods of only 2.35 and 2.18 days, respectively. The stars in these binaries are rapid rotators (consistent with synchronous rotation maintained by tidal forces): V1061 Cyg Ab has a measured $v \sin i=20 \pm 3 \mathrm{~km} \mathrm{~s}^{-1}(\S 2.1)$, and FL Lyr B has $v \sin i=25 \pm 2 \mathrm{~km} \mathrm{~s}^{-1}$ (Popper et al. 1986). Both binaries are strong ROSAT X-ray sources (Voges et al. 1999), and their X-ray luminosities (Table 12) are in line with those of active single and binary stars with similar $v \sin i$ values (e.g., Cutispoto et al. 2003, Fig. 5). Although to our knowledge no observations exist to verify whether the $\mathrm{Ca}$ II $\mathrm{H}$ and $\mathrm{K}$ lines are in emission, both systems show signs of intrinsic variability in the light curve, suggesting the presence of spots (see Popper et al. [1986] for FL Lyr and $\S 2.3$ for V1061 Cyg). They are thus active binaries. RW Lac and HS Aur, on the other hand, do not show a radius discrepancy and happen to have much longer orbital periods of 10.37 and 9.82 days, respectively, and their components are slow rotators. No Ca II emission is seen in HS Aur (Popper 1976). Neither binary was detected as an X-ray source by ROSAT (Voges et al. 1999), and by all accounts they appear inactive. A direct relation is thus seen for stars of the same mass between the activity level and the increased stellar size compared to predictions from standard models (i.e., those adopting a mixing-length parameter matching the Sun): active stars are larger, and inactive ones appear normal. The evidence for V1061 Cyg and other later type stars also indicates that active stars are cooler.

This seems to have been mentioned only as a possibility (among several others) in previous studies reporting radius discrepancies for stars under a solar mass, at least those originating from the eclipsing binary community. But as a matter of fact, the connection between activity and the global properties of low-mass stars has been studied in some detail previously in a slightly different context, although without direct and precise knowledge of the masses and radii of the stars involved. Mullan \& MacDonald (2001) investigated the effects of magnetic fields on the sizes and effective temperatures of active versus inactive $M$ dwarfs and found empirical evidence that a higher activity level leads to larger radii and cooler temperatures. Their sample consisted of single stars with effective temperature determinations from infrared spectroscopy and bolometric luminosities from multiband photometry, from which stellar radii were inferred indirectly with typical uncertainties of $10 \%-15 \%$. Although their work focused mainly on the consequences for the internal structure of fully convective stars, their initial attempts at modeling magnetic fields were successful in describing these effects to first order. The present study on V1061 Cyg shows the activity-radius connection clearly for stars with accurately determined dynamical masses, radii, and temperatures good to $1 \%-3 \%$. More importantly, the effect is seen for objects that are only $7 \%$ less massive than the Sun.

A theoretical understanding of this connection is also not new and provides some insight into the better agreement between the observations for V1061 Cyg and the low- $\alpha_{\mathrm{ML}}$ models in Figure 14. Strong magnetic fields commonly associated with chromospheric activity have been shown to inhibit the efficiency of convective heat transport (e.g., Bray \& Loughhead 1964, p. 271; Gough \& Tayler 1966; Stein et al. 1992 and references therein), 
TABLE 12

Parameters for Eclipsing Systems in the Mass Range of V1061 Cyg

\begin{tabular}{|c|c|c|c|c|c|c|c|c|}
\hline Star & $\begin{array}{l}\text { Mass } \\
\left(M_{\odot}\right)\end{array}$ & $\begin{array}{l}\text { Radius } \\
\left(R_{\odot}\right)\end{array}$ & $\begin{array}{l}\log g \\
\text { (cgs) }\end{array}$ & $\begin{array}{l}T_{\text {eff }} \\
(\mathrm{K})\end{array}$ & $\begin{array}{c}M_{V} \\
(\mathrm{mag})\end{array}$ & $\begin{array}{l}\text { Period } \\
\text { (days) }\end{array}$ & $\begin{array}{c}v \sin i^{\mathrm{a}} \\
\left(\mathrm{km} \mathrm{s}^{-1}\right)\end{array}$ & $\begin{array}{c}\log L_{\mathrm{X}}^{\mathrm{b}} \\
\left(\mathrm{ergs} \mathrm{s}^{-1}\right)\end{array}$ \\
\hline V1061 Cyg Aa............ & $1.282 \pm 0.015$ & $1.615 \pm 0.017$ & $4.129 \pm 0.011$ & $6180 \pm 100$ & $3.456 \pm 0.074$ & 2.3467 & $36 \pm 2$ & 30.12 \\
\hline V1061 Cyg B ${ }^{\mathrm{c}} \ldots \ldots \ldots \ldots$ & $0.925 \pm 0.036$ & $0.870 \pm 0.087$ & $4.525 \pm 0.088$ & $5670 \pm 150$ & $5.23 \pm 0.16$ & $\ldots$ & $2 \pm 3$ & $\ldots$ \\
\hline FL Lyr A....................... & $1.221 \pm 0.016$ & $1.282 \pm 0.028$ & $4.309 \pm 0.020$ & $6150 \pm 100$ & $3.95 \pm 0.09$ & 2.1782 & $30 \pm 2$ & 30.19 \\
\hline FL Lyr B ......................... & $0.960 \pm 0.012$ & $0.962 \pm 0.028$ & $4.454 \pm 0.026$ & $5300 \pm 100$ & $5.37 \pm 0.10$ & 2.1782 & $25 \pm 2$ & \\
\hline HS Aur A & $0.900 \pm 0.019$ & $1.004 \pm 0.024$ & $4.389 \pm 0.023$ & $5350 \pm 75$ & $5.23 \pm 0.08$ & 9.8154 & $\ldots$ & $<29.2$ \\
\hline HS Aur B ...................... & $0.879 \pm 0.017$ & $0.873 \pm 0.024$ & $4.500 \pm 0.025$ & $5200 \pm 75$ & $5.68 \pm 0.08$ & 9.8154 & $\ldots$ & $\ldots$ \\
\hline $\operatorname{Sun}^{\mathrm{d}} \ldots \ldots \ldots \ldots \ldots \ldots \ldots \ldots$ & 1.000 & 1.000 & 4.438 & 5780 & 4.83 & $\ldots$ & 2 & $26.4-27.7$ \\
\hline
\end{tabular}

${ }^{\text {a }}$ Measured rotational velocities for the eclipsing systems are all consistent with synchronous rotation. Rotational velocity measurements for HS Aur are not available, but Popper et al. (1986) have shown that the lines are sharp. Synchronous velocities for the primary and secondary are 5.2 and $4.5 \mathrm{~km} \mathrm{~s}{ }^{-1}$, respectively.

b The values listed are for each system as a whole.

${ }^{c}$ Values for the radius and $\log g$ are inferred from other properties; see $\S 5.3$.

d The range in the X-ray luminosity of the Sun represents the change during the activity cycle (Peres et al. 2000).
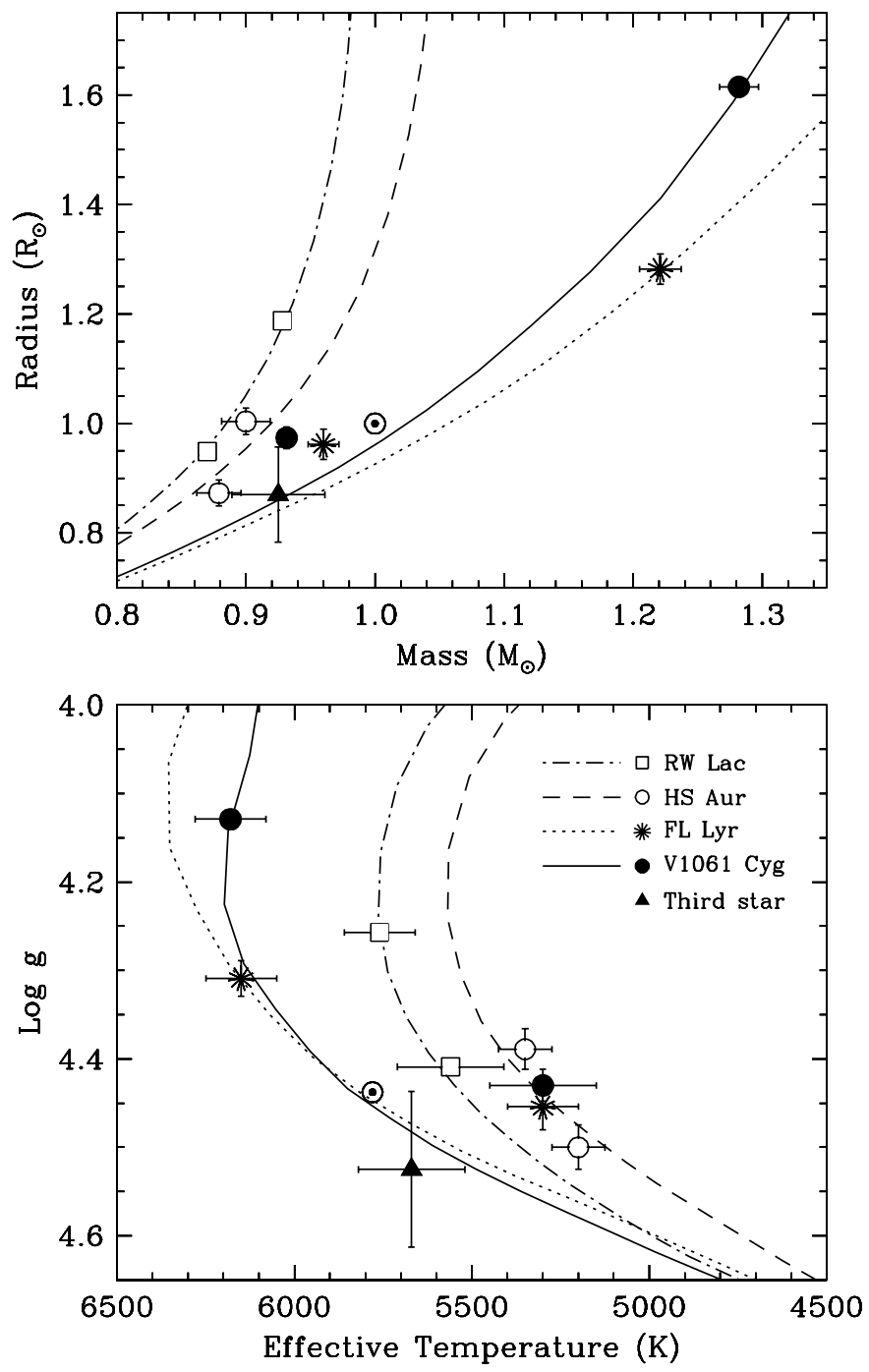

FIG. 15.-Properties of V1061 Cyg and three other eclipsing binaries having one star closely matching V1061 Cyg Ab in mass, compared against the YonseiYale models by Yi et al. (2001) and Demarque et al. (2004). For each binary system the model isochrone best fitting all observed properties is shown: $Z=0.0118$ and age $=10.7$ Gyr for RW Lac, $Z=0.024$ and age $=10.5$ Gyr for HS Aur, $Z=$ 0.026 and age $=2.4 \mathrm{Gyr}$ for FL Lyr, and $Z=Z_{\odot}$ and age $=3.4 \mathrm{Gyr}$ for V1061 Cyg. The secondary star in FL Lyr is significantly larger than predicted, as is V1061 Cyg Ab (see text). The Sun (circled dot) is also shown for reference, along with the third component of V1061 Cyg (§ 5.3). and as a result the size of the star must grow larger to radiate away the same amount of energy. The decreased convection effectively leads to a lower value of the mixing-length parameter (see Tayler 1987), which explains the better fit to the radius of the secondary using the Baraffe et al. (1998) models with $\alpha_{\mathrm{ML}}=1.0$ as opposed to those with the solar value of $\alpha_{\mathrm{ML}}=1.9$. Similar improvements in the fit to other low-mass stars using a reduced mixing-length parameter were also reported by Clausen et al. (1999a) and in fact much earlier by Gabriel (1969) and Cox et al. (1981).

The enhanced activity in V1061 Cyg Ab is driven by rapid (synchronous) rotation induced by tidal forces. The primary star is rotating even more rapidly ( $v \sin i=36 \pm 2 \mathrm{~km} \mathrm{~s}^{-1}$ ), yet it shows no obvious indication of a significantly larger radius compared to standard models. This is most likely because it is a more massive star (spectral type F9, $M=1.282 M_{\odot}$ ) and therefore its convective envelope (where magnetic activity takes place) is significantly reduced. To illustrate this in a more quantitative way, Figure 16 shows the mass of the convective envelope for mainsequence stars as a function of stellar mass from the models by Siess et al. (1997; see also Siess et al. 2000), for solar composition and the age of 3.4 Gyr we infer for the system. In the bottom panel the envelope mass is expressed as a percentage of the stellar mass. The inset shows an enlargement of the region relevant to V1061 Cyg. According to these models, the convective envelope of the secondary represents about $4.7 \%$ of its total mass, whereas that of the primary is only $0.2 \%(\sim 20$ times smaller $)$. A similarly reduced convective envelope for the primary of FL Lyr $(M=$ $1.221 M_{\odot}$ ) explains why that star also does not show evidence of an enlarged radius, while the secondary (which is similar in mass to V1061 Cyg Ab) does.

The extent to which a star is enlarged by this effect may be expected to depend on the strength of the activity. We note, for instance, that although models with $\alpha_{\mathrm{ML}}=1.0 \mathrm{seem}$ to match the radius of V1061 Cyg Ab better, the same models still do not reproduce the properties of apparently more active lower mass stars such as YY Gem (Torres \& Ribas 2002), CU Cnc (Ribas 2003), GU Boo (López-Morales \& Ribas 2005), and others. It is clear that further examples of stars (both active and inactive) in the suitable mass range with accurately determined parameters are needed to explore this. Systems such as V1061 Cyg and FL Lyr, with components of appreciably different mass and primaries that are larger than $\sim 1.2 M_{\odot}$, are particularly useful because they allow the activity-radius effect to be separated out, since the primary should not be significantly affected. 

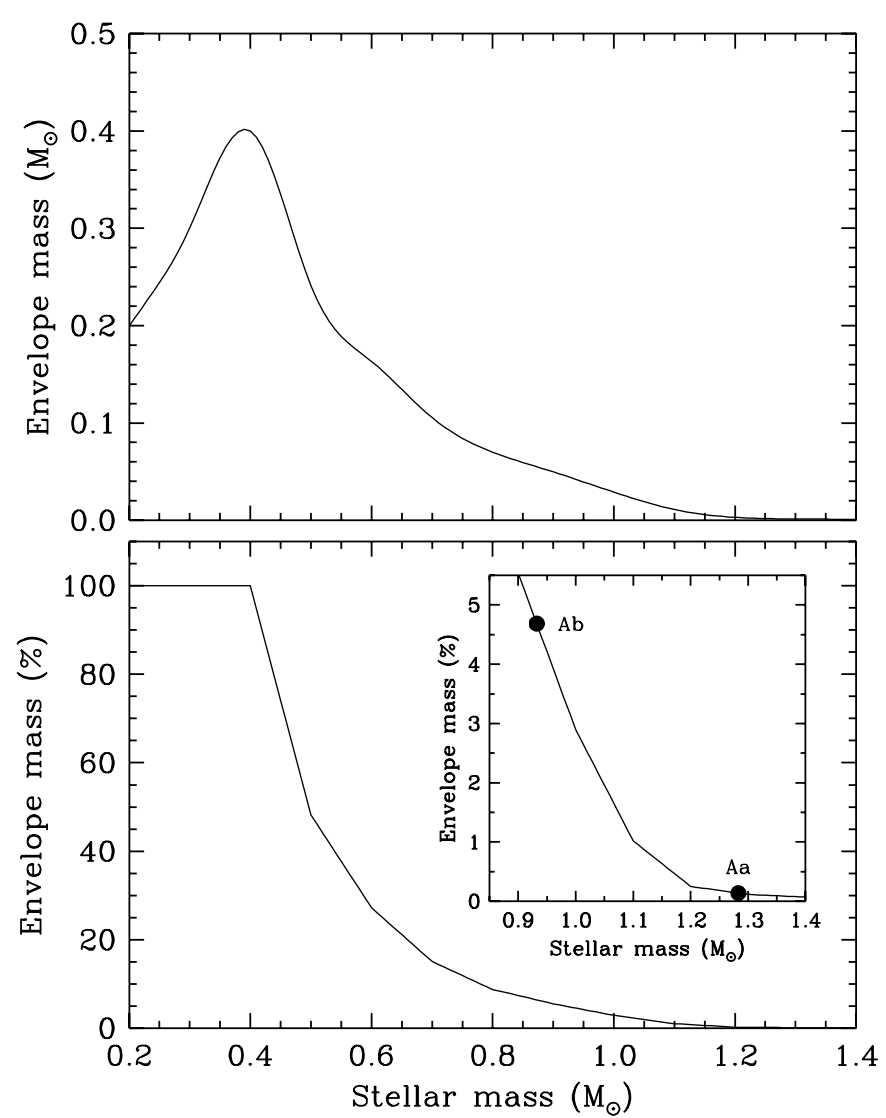

FIG. 16.-Mass of the convective envelope for main-sequence stars as a function of stellar mass, based on evolution models by Siess et al. $(1997,2000)$ for solar composition and the estimated age for V1061 Cyg of 3.4 Gyr. The envelope mass is shown in solar masses (top) and as a fraction of the stellar mass (bottom). According to these models, stars of $0.4 M_{\odot}$ and lower are fully convective. In the inset the region around the masses of V1061 Cyg is shown on a larger scale, and the envelope mass of both stars is indicated.

\subsection{The Tertiary Star}

The V1061 Cyg system is especially valuable for containing a third star of mass indistinguishable from that of the active star $\mathrm{Ab}$, but with slow rotation. It is presumably an inactive analog of the secondary, with the same age and chemical composition, and is thus ideal for testing the effect discussed above. We do not have a direct measurement of its size, but the radius can be inferred from the effective temperature and luminosity and is $R_{\mathrm{B}}=$ $0.870 \pm 0.087 R_{\odot}$. While this is formally smaller than the secondary radius of $R_{\mathrm{Ab}}=0.974 \pm 0.020 R_{\odot}$, as we expected, it is not precise enough for a definitive test. Other inferred properties of the tertiary are listed in Table 11. The star is represented graphically in Figure 15, where it is seen to be in good agreement with the isochrone for the primary, within the admittedly large errors. The effective temperature we determine for the tertiary $(5670 \pm 150 \mathrm{~K})$ is somewhat hotter than that of the secondary even though the masses are similar, which again is consistent with our conclusion that the activity in the secondary has made that star cooler.

The formal uncertainty in $R_{\mathrm{B}}$ is dominated by errors in the light fraction $l_{\mathrm{B}}$ and effective temperature $T_{\mathrm{eff}}^{\mathrm{B}}$, in that order of importance. Improvements in the former could be made, for example, by direct detection of the third star and measurement of its brightness through speckle interferometry or adaptive optics imaging ( $(4)$. A better temperature estimate would require spectroscopy with higher $\mathrm{S} / \mathrm{N}$, although the triple-lined nature of the spectrum and the faintness of the star would still pose a chal- lenge. Additional radial velocity measurements over the coming years covering the next nodal passage in the outer orbit (in 2009.1) would also be important to refine the value of the tertiary mass.

\section{CONCLUDING REMARKS}

The results of our spectroscopic, photometric, and astrometric analyses of the V1061 Cyg system have taken us in a rather different direction than we anticipated when we began this study. The possible status of the object as an example of the rare class of cool Algols is now clearly ruled out. Instead, we have shown here that it is a hierarchical triple system with an outer period of $15.8 \mathrm{yr}$, in which the eclipsing inner pair is well detached, is composed of main-sequence stars, has a mass ratio quite different from unity, and has a secondary that is slightly below a solar mass. The absolute masses and radii for the binary components are determined with a relative precision of $2 \%$ or better, and the mass of the third star is good to $4 \%$.

While the primary star is well fitted in mass, radius, temperature, and luminosity by standard stellar evolution models with a metallicity near solar and a mixing-length parameter set by the calibration to the Sun, the secondary appears $\sim 10 \%$ too large. This discrepancy is 5 times the size of the observational errors and quite surprising for a star that differs by only $7 \%$ in mass from the Sun. There are also indications that it is cooler than predicted by some $200 \mathrm{~K}$. V1061 Cyg is yet another example highlighting our incomplete understanding of the structure and evolution of stars in the lower main sequence. Similar differences in size and temperature have been noticed previously for lower mass stars with accurately measured properties, but the source of the problem has remained unclear. By comparing V1061 Cyg Ab to several other objects of nearly the same mass, we have identified the activity level as a key factor distinguishing cases that show the radius discrepancy from those that do not. This link between activity and increased radius has been mentioned in the literature before but is shown here for the first time for stars with accurately known masses, radii, and temperatures.

It is often stated that the structure and evolution of stars (particularly those close to a solar mass) are completely determined once the chemical composition and mass are specified. It is quite clear now that for stars of order $1 M_{\odot}$ or less an additional parameter must be taken into account, which has to do with the level of chromospheric activity. Whether this parameter is directly the rotational speed (e.g., $v \sin i$ ) or period, the magnetic field strength, the Rossby number (e.g., Noyes et al. 1984; Basri 1987 ), or some other more complicated activity indicator remains to be determined. To first order it appears that the effective mixing length may be a useful proxy, but with one exception current stellar evolution models are not publicly available for more than one value of $\alpha_{\mathrm{ML}}$, so testing this is somewhat difficult in practice. If the predictions from the models are to reach an accuracy matching current observations of low-mass stars ( $\sim 1 \%-2 \%$ relative errors in the masses and radii), this effect can no longer be ignored. Further progress will require more examples of binary systems with well-determined physical parameters and different levels of activity in the relevant mass regime in order to help calibrate any such parameter.

Testing models of single-star evolution by means of eclipsing binaries, as astronomers have done for decades, might perhaps be seen as part of the problem since the enhanced activity displayed by many of these systems is a direct result of tidal synchronization that occurs only in close binaries. Although wider eclipsing pairs with inactive components under $1 M_{\odot}$ certainly do exist, they are less common and in some respects more difficult to 
study. Stars near the bottom of the main sequence, $M$ dwarfs in particular, are found to be active more often than not.

The effects of magnetic fields on the evolution of stars have already begun to be explored by theorists (e.g., D'Antona et al. 2000; Mullan \& MacDonald 2001; Maeder \& Meynet 2003; although the latter authors focus on more massive stars), and initial comparisons with observations are encouraging. One area where this is likely to have a significant impact is the study of T Tauri stars, which are typically very active. Even in substellar objects such as brown dwarfs activity appears to be quite common and might be expected to have similar consequences on their structure. Some evidence for this has already been reported (Mohanty et al. 2004).

We are grateful to J. Andersen, P. Berlind, M. Calkins, J. Caruso, D. W. Latham, R. P. Stefanik, and J. Zajac for their efforts at the telescope to obtain the majority of the spectroscopic observations for V1061 Cyg used in this work, as well as to R. J. Davis for maintaining the CfA echelle database. The referee, J. Andersen, is thanked for a number of insightful comments and suggestions that improved the original manuscript. We also thank J. M. Kreiner for providing unpublished times of eclipse for the binary, as well as R. Neuhäuser and B. Stelzer for assistance with the X-ray observations. G. T. acknowledges partial support for this work from NSF grant AST 04-06183 and NASA's MASSIF SIM Key Project (BLF57-04). L. M. and H. S. were supported by Gettysburg College and the Delaware Space Grant Consortium. Additional thanks go to Peter Mack and Gary Hummer for technical support at the Gettysburg College Observatory. This research has made use of the SIMBAD database, operated at CDS, Strasbourg, France, and of NASA's Astrophysics Data System Abstract Service. This work makes use of data products from the Two Micron All Sky Survey, which is a joint project of the University of Massachusetts and the Infrared Processing and Analysis Center/California Institute of Technology, funded by NASA and the NSF.
Agerer, F., \& Hübscher, J. 1995, Inf. Bull. Variable Stars, 4222, 1 1996, Inf. Bull. Variable Stars, 4383, 1

Alencar, S. H. P., \& Vaz, L. P. R. 1997, A\&A, 326, 257

Al-Naimiy, H. M. 1978, Ap\&SS, 53, 181

Alonso, A., Arribas, S., \& Martínez-Roger, C. 1996, A\&A, 313, 873

Andersen, J. 1991, A\&A Rev., 3, 91

1997, in IAU Symp. 189, Fundamental Stellar Properties: The Inter-

action between Observation and Theory, ed. T. R. Bedding, A. J. Booth, \&

J. Davis (Dordrecht: Reidel), 99

. 2003, Proc. SPIE, 4838, 466

Baraffe, I., Chabrier, G., Allard, F., \& Hauschildt, P. H. 1998, A\&A, 337, 403

Basri, G. 1987, ApJ, 316, 377

Bray, R. J., \& Loughhead, R. E. 1964, Sunspots (New York: Dover)

Chabrier, G., \& Baraffe, I. 1997, A\&A, 327, 1039

Claret, A. 1998, A\&AS, 131, 395

Clausen, J. V., Baraffe, I., Claret, A., \& VandenBerg, D. A. 1999a, in ASP Conf. Ser. 173, Theory and Tests of Convection in Stellar Structure, ed. A. Giménez, E. F. Guinan, \& B. Montesinos (San Francisco: ASP), 265

Clausen, J. V., Helt, B., \& Olsen, E. H. 1999b, in ASP Conf. Ser. 173, Theory and Tests of Convection in Stellar Structure, ed. A. Giménez, E. F. Guinan, \& B. Montesinos (San Francisco: ASP), 321

Cox, A. N. 2000, Allen's Astrophysical Quantities (4th ed.; Berlin: Springer)

Cox, A. N., Shaviv, G., \& Hodson, S. W. 1981, ApJ, 245, L37

Creevey, O. L., et al. 2005, ApJ, 625, L127

Cutispoto, G., Tagliaferri, G., de Medeiros, J. R., Pastori, L., Pasquini, L., \& Andersen, J. 2003, A\&A, 397, 987

D’Antona, F., Ventura, P., \& Mazzitelli, I. 2000, ApJ, 543, L77

Dawson, P. C., \& De Robertis, M. M. 2004, AJ, 127, 2909

Demarque, P., Woo, J.-H., Kim, Y.-C., \& Yi, S. K. 2004, ApJS, 155, 667

Díaz-Cordovés, J., Claret, A., \& Giménez, A. 1995, A\&AS, 110, 329

Diethelm, R. 1986, BBSAG Bull., 81, 3

Eggenberger, P., Charbonnel, C., Talon, S., Meynet, G., Maeder, A., Carrier, F., \& Bourban, G. 2004, A\&A, 417, 235

ESA 1997, The Hipparcos and Tycho Catalogues (ESA SP-1200; Noordwijk: ESA)

Etzel, P. B. 1981, in Photometric and Spectroscopic Binary Systems, ed. E. B. Carling \& Z. Kopal (NATO ASI Ser. C., 69; Dordrecht: Reidel), 111

Fekel, F. C., Jr. 1981, ApJ, 246, 879

Fitzpatrick, E. L., Ribas, I., Guinan, E. F., Maloney, F. P., \& Claret, A. 2003, ApJ, 587, 685

Gabriel, M. 1969, in Low-Luminosity Stars, ed. S. S. Kumar (New York: Gordon \& Breach), 267

Girardi, L., Bressan, A., Bertelli, G., \& Chiosi, C. 2000, A\&AS, 141, 371

Gough, D. O., \& Tayler, R. J. 1966, MNRAS, 133, 85

Guinan, E. F., et al. 1998, ApJ, 509, L21

Høg, E., et al. 2000, A\&A, 355, L27

Hoxie, D. T. 1973, A\&A, 26, 437

Irwin, J. B. 1952, ApJ, 116, 211

Johnson, H. L., \& Morgan, W. W. 1953, ApJ, 117, 313

Kreiner, J. M., Kim, C. H., \& Nha, I. S. 2001, An Atlas of $O-C$ Diagrams of

Eclipsing Binary Stars (Krakow: Wydawnctwo Naukowe Ap)

Lacy, C. H. 1977, ApJS, 34, 479

1992, AJ, 104, 801
REFERENCES

Lacy, C. H. S. 2001, Inf. Bull. Variable Stars, 5067, 1

2002, Inf. Bull. Variable Stars, 5357, 1

2003, Inf. Bull. Variable Stars, 5487, 1

2004, Inf. Bull. Variable Stars, 5577, 1

Lacy, C. H. S., Claret, A., \& Sabby, J. A. 2004, AJ, 128, 1840

Lacy, C. H. S., Straughn, A., \& Denger, F. 2002, Inf. Bull. Variable Stars, 5251, 1

Lacy, C. H. S., Torres, G., Claret, A., \& Vaz, L. P. R. 2005, AJ, 130, 2838

Lastennet, E., Fernandes, J., Valls-Gabaud, D., \& Oblak, E. 2003, A\&A, 409, 611

Latham, D. W. 1992, in IAU Colloq. 135, Complementary Approaches to Double and Multiple Star Research, ed. H. A. McAlister \& W. I. Hartkopf (ASP Conf. Ser. 32; San Francisco: ASP), 110

Latham, D. W., Nordström, B., Andersen, J., Torres, G., Stefanik, R. P., Thaller, M., \& Bester, M. 1996, A\&A, 314, 864

Latham, D. W., Stefanik, R. P., Torres, G., Davis, R. J., Mazeh, T., Carney, B. W., Laird, J. B., \& Morse, J. A. 2002, AJ, 124, 1144

Lejeune, Th., Cuisinier, F., \& Buser, R. 1998, A\&AS, 130, 65

López-Morales, M., \& Ribas, I. 2005, ApJ, 631, 1120

Ludwig, H. G., Freytag, F., \& Steffen, M. 1999, A\&A, 346, 111

Maeder, A., \& Meynet, G. 2003, A\&A, 411, 543

Mohanty, S., Jayawardhana, R., \& Basri, G. 2004, ApJ, 609, 885

Mullan, D. J., \& MacDonald, J. 2001, ApJ, 559, 353

Nordström, B., Latham, D. W., Morse, J. A., Milone, A. A. E., Kurucz, R. L., Andersen, J., \& Stefanik, R. P. 1994, A\&A, 287, 338

Noyes, R. W., Hartmann, L. W., Baliunas, S. L., Duncan, D. K., \& Vaughan, A. H. 1984, ApJ, 279, 763

Ogłoza, W., Dróżdż, M., \& Zoła, S. 2000, Inf. Bull. Variable Stars, 4877, 1

Peres, G., Orlando, S., Reale, F., Rosner, R., \& Hudson, H. 2000, ApJ, 528, 537

Popper, D. M. 1976, Inf. Bull. Variable Stars, 1201, 1

1980, ARA\&A, 18, 115

1992, in IAU Symp. 151, Evolutionary Processes in Interacting Binary

Stars, ed. Y. Kondo, R. F. Sisteró, \& R. S. Polidan (Dordrecht: Kluwer), 395 1994, AJ, 108, 1091

1996, ApJS, 106, 133

1997, AJ, 114, 1195

Popper, D. M., \& Etzel, P. B. 1981, AJ, 86, 102

Popper, D. M., Lacy, C. H., Frueh, M. L., \& Turner, A., E. 1986, AJ, 91, 383

Pourbaix, D., \& Jorissen, A. 2000, A\&AS, 145, 161

Press, W. H., Teukolsky, S. A., Vetterling, W. T., \& Flannery, B. P. 1992,

Numerical Recipes in FORTRAN (2nd. ed.; Cambridge: Cambridge Univ. Press)

Ramírez, I., \& Meléndez, J. 2005, ApJ, 626, 465

Ribas, I. 2003, A\&A, 398, 239

Sheets, H. A., Marschall, L. A., \& Torres, G. 2003, BAAS, 202, 08.05

Siess, L., Dufour, E., \& Forestini, M. 2000, A\&A, 358, 593

Siess, L., Forestini, M., \& Dougados, C. 1997, A\&A, 324, 556

Stein, R. F., Brandenburg, A., \& Nordlund, A. 1992, in ASP Conf. Ser. 26, Seventh Cambridge Workshop on Cool Stars, Stellar Systems, and the Sun, ed. M. S. Giampapa \& J. A. Bookbinder (San Francisco: ASP), 148

Strohmeier, W., \& Knigge, R. 1959, Veröff. Remeis-Sternw. Bamberg, 5, 4

Strohmeier, W., Knigge, R., \& Ott, H. 1962, Veröff. Remeis-Sternw. Bamberg, 5,14 
Tayler, R. J. 1987, MNRAS, 227, 553

Torres, G., Andersen, J., Nordström, B., \& Latham, D. W. 2000, AJ, 119, 1942

Torres, G., \& Ribas, I. 2002, ApJ, 567, 1140

Torres, G., Stefanik, R. P., Andersen, J., Nordström, B., Latham, D. W., \& Clausen, J. V. 1997, AJ, 114, 2764

Trampedach, R., Stein, R. F., Christensen-Dalsgaard, J., \& Nordlund, Å. 1999, in ASP Conf. Ser. 173, Theory and Tests of Convection in Stellar Structure, ed. A. Giménez, E. F. Guinan, \& B. Montesinos (San Francisco: ASP), 233 van Leeuwen, F., \& Evans, D. W. 1998, A\&AS, 130, 157 Voges, W., et al. 1999, A\&A, 349, 389

Wade, R. A., \& Rucinski, S. M. 1985, A\&AS, 60, 471

Yi, S. K., Demarque, P., Kim, Y.-C., Lee, Y.-W., Ree, C. H., Lejeune, T., \& Barnes, S. 2001, ApJS, 136, 417

Zucker, S., \& Mazeh, T. 1994, ApJ, 420, 806

Zucker, S., Torres, G., \& Mazeh, T. 1995, ApJ, 452, 863 